\title{
MANIFOLDS OF HILBERT SPACE PROJECTIONS
}

\author{
R. H. LEVENE AND S. C. POWER
}

\begin{abstract}
The Hardy space $H^{2}(\mathbb{R})$ for the upper half plane together with a multiplicative group of unimodular functions $u(\lambda)=$ $\exp \left(i\left(\lambda_{1} \psi_{1}+\cdots+\lambda_{n} \psi_{n}\right)\right), \lambda \in \mathbb{R}^{n}$, gives rise to a manifold $\mathcal{M}$ of orthogonal projections for the subspaces $u(\lambda) H^{2}(\mathbb{R})$ of $L^{2}(\mathbb{R})$. For classes of admissible functions $\psi_{i}$ the strong operator topology closures of $\mathcal{M}$ and $\mathcal{M} \cup \mathcal{M}^{\perp}$ are determined explicitly as various $n$-balls and $n$-spheres. The arguments used are direct and rely on the analysis of oscillatory integrals (Stein [17]) and Hilbert space geometry. Some classes of these closed projection manifolds are classified up to unitary equivalence. In particular the FourierPlancherel 2-sphere and the hyperbolic 3-sphere of Katavolos and Power [8] appear as distinguished special cases admitting nontrivial unitary automorphism groups which are explicitly described.
\end{abstract}

\section{INTRODUCTION}

Let $\mathcal{M}$ be a set of closed subspaces of a Hilbert space $\mathcal{H}$ endowed with the strong operator topology inherited from the identification of closed subspaces $\mathcal{K}$ with their self-adjoint projections $[\mathcal{K}]: \mathcal{H} \rightarrow \mathcal{K}$. If $\mathcal{M}$ is finitely parametrised in the sense that

$$
\mathcal{M}=\left\{\left[\mathcal{K}_{\lambda}\right]: \lambda \in M \subseteq \mathbb{R}^{n}\right\}
$$

with $M$ a topological manifold, then $\mathcal{M}$ may in fact be homeomorphic to $M$. Furthermore $\mathcal{M}$ may admit a certain local unitary description and an associated smooth structure under which it is a diffeomorph of a differentiable manifold in $\mathbb{R}^{n}$. Natural examples of such manifolds of projections are provided by Grassmannian manifolds and their submanifolds. Also, operators $T$ in the first Cowen-Douglas class [1], [2] for a complex connected domain $\Omega$ in $\mathbb{C}^{m}$ provide diverse realisations (even for $m=1$ ) of domains in $\mathbb{R}^{2 m}$, namely

$$
\mathcal{M}_{T}=\left\{[\operatorname{ker}(T-\omega I)]: \omega \in \Omega \subseteq \mathbb{R}^{2 m}\right\} .
$$

However our primary motivating examples derive from invariant subspaces for semigroups of unitary operators, such as the semigroup $\mathcal{W}=\left\{\alpha U_{t} V_{s}: s, t \in \mathbb{R}_{+},|\alpha|=1\right\}$ associated with jointly irreducible one parameter unitary groups satisfying the Weyl commutation relations. Furthermore, such subspaces and their complementary spaces

May 13, 2009.

2000 Mathematics Subject Classification. 47B38, 46E20, 58D15.

Key words and phrases. Lie semigroups, projection manifolds, Hardy spaces. 
are generally infinite dimensional. These examples motivate the consideration of general subspace manifolds as formulated in Definitions 2.1, 2.2 and 2.3 .

The embracing realm we consider is the set of Hilbert space subspaces of the form

$$
u H^{2}(\mathbb{R}), \quad u \overline{H^{2}(\mathbb{R})}, \quad L^{2}(E)
$$

where $H^{2}(\mathbb{R})$ is the Hardy space for the upper half plane, $u(x)$ is unimodular, and $L^{2}(E)$ is the space of functions supported on a measurable set $E$. These spaces are fundamental in the analysis of various shift invariant subspaces. They feature, in transposed form, in Beurling's celebrated characterisation of invariant subspaces for the shift operator on $H^{2}(\mathbb{T})$ as well as in many other operator function theory perspectives. See Nikolskii [13] for example. We examine subspace manifolds of the form

$$
\mathcal{M}=\mathcal{M}(\mathcal{S})=\left\{\left[e^{i \psi(x)} H^{2}(\mathbb{R})\right]: \psi(x) \in \mathcal{S}\right\},
$$

where $\mathcal{S}$ is a finite dimensional real vector space of real-valued functions, we analyse limits of projections and we identify the associated closed topological manifolds. Our approach and the ensuing identifications give a unified explanation for various so called "strange limits" of projections. These include the special cases considered by Katavolos and Power [7], [8] which were derived by ad hoc arguments leaning on operator algebra methods. Specifically we show by direct methods that the space of functions

$$
\mathcal{S}_{1}=\left\{\lambda_{1} x+\lambda_{2} x^{2}: \lambda=\left(\lambda_{1}, \lambda_{2}\right) \in \mathbb{R}^{2}\right\},
$$

has subspace manifold $\mathcal{M}\left(\mathcal{S}_{1}\right)$ whose closure is homeomorphic to the closed unit disc, while for the space

$$
\mathcal{S}_{2}=\left\{\lambda_{1} \log |x|+\lambda_{2} x+\lambda_{3} x^{-1}: \lambda \in \mathbb{R}^{3}\right\},
$$

the manifold $\mathcal{M}\left(\mathcal{S}_{2}\right)$ has closure homeomorphic to the closed unit ball in $\mathbb{R}^{3}$. In contrast, the closures of Cowen-Douglas projection manifolds are generally one point compactifications.

A consequence of the limit projection analyses in [7], [8] is that a reducing invariant subspace for the Weyl semigroup $\mathcal{W}$, or for the $a x+b$ unitary semigroup (with $a \geq 1$ and $b \geq 0$ ), turns out to be a strong operator topology limit of a sequence of purely invariant projections, that is, a limit of those with no reducing part. We obtain in Theorem $3.7 \mathrm{a}$ similar phenomenon for the multiplication semigroup $\left\{M_{e^{i \lambda x}}: \lambda \geq 0\right\}$ acting on $L^{2}(\mathbb{R})$. Equivalently, translating to the circle $\mathbb{T}$, we show that each reducing invariant subspace for the bilateral shift, which corresponds to a measurable subset of $\mathbb{T}$, is a strong operator topology limit of Beurling projections $\left[u H^{2}(\mathbb{T})\right]$. We are not aware of any other proof, direct or indirect, of this seemingly classical fact.

Our principal tool is Theorem 3.4 which, for a function $\psi$ in a certain admissible class, identifies the limit of the projections $\left[e^{i n \psi} H^{2}(\mathbb{R})\right]$, as 
$n \rightarrow \infty$, with the projection $\left[L^{2}\left(\left(\psi^{\prime}\right)^{-1}(-\infty, 0)\right)\right]$. The proof makes use of methods of Stein [17] for the analysis of oscillatory integrals. We go on to show that for quite general $n$ dimensional spaces $\mathcal{S}$ of admissible functions the subspace manifold $\mathcal{M}(\mathcal{S})$ has closure homeomorphic to the closed unit ball in $\mathbb{R}^{n}$. Moreover, in these cases the two-component subspace manifold $\mathcal{M}(\mathcal{S}) \cup \mathcal{M}(\mathcal{S})^{\perp}$ has closure, denoted $\Sigma(\mathcal{S})$, which is homeomorphic to an $n$-sphere. The 2-sphere $\Sigma_{\mathrm{FP}}=\Sigma\left(\mathcal{S}_{1}\right)$ is the so-called Fourier-Plancherel sphere of [8]. (See Figure 5.1 and Example 4.9.) It is natural to consider how such Hilbert space manifolds may be classified geometrically, that is, up to unitary equivalence, and in the examples of Section 4 and Section 5.3 we distinguish a number of distinct 2-spheres and 3-spheres.

In Section 5 we consider the sphere $\Sigma\left(\mathcal{S}_{1}\right)$ and its hyperbolic variant, the 3 -sphere $\Sigma\left(\mathcal{S}_{2}\right)$, from the point of view of their unitary automorphism symmetries. The analysis here exploits the nontrivial foliations induced by the natural order on projections. Let $F: L^{2}(\mathbb{R}) \rightarrow L^{2}(\mathbb{R})$ be the Fourier-Plancherel transform, that is, the unitary operator

$$
F f(x)=\frac{1}{\sqrt{2 \pi}} \int_{-\infty}^{\infty} f(y) e^{-i x y} d y, \quad f \in L^{2}(\mathbb{R}) .
$$

It is shown that the group $\mathcal{U}\left(\Sigma\left(\mathcal{S}_{1}\right)\right)$ of unitaries which act bijectively on $\Sigma\left(\mathcal{S}_{1}\right)$ is generated by the set

$$
\left\{M_{e^{i \lambda x}}, M_{e^{i s x^{2}}}, \alpha I, F: \lambda, s \in \mathbb{R},|\alpha|=1\right\}
$$

consisting of two 1-parameter unitary groups, the scalar circle group and $F$. In particular, this group contains the group of dilations $\left\{V_{t}\right.$ : $t \in \mathbb{R}\}$, as we show explicitly in Lemma 5.1. A similarly detailed description is given for $\mathcal{U}\left(\Sigma\left(\mathcal{S}_{2}\right)\right)$ and this leads to the identification of the unitary automorphism group as a certain double semidirect product

$$
\operatorname{Ad}\left(\mathcal{U}\left(\Sigma\left(\mathcal{S}_{2}\right)\right)\right) \cong\left(\mathbb{R}^{3} \rtimes \mathbb{R}\right) \rtimes(\mathbb{Z} / 2 \mathbb{Z})^{2} .
$$

The manifolds $\mathcal{M}(\mathcal{S})$, Beurling subspace manifolds in our terminology, may be regarded as smooth in the strict, locally unitary, sense that a neighbourhood of a subspace $\mathcal{K}$ is given by the local action on $\mathcal{K}$ of a certain unitary group representation of $\mathbb{R}^{n}$. Furthermore the Fourier-Plancherel 2-sphere $\Sigma_{\mathrm{FP}}$ is remarkable in being smooth in this way at all points except the poles $0, I$. It of interest then to identify similar compact projection manifolds which are smooth off a finite set. In this regard we see in Section 5.3 that this is not generally the case for other polynomial 2-spheres $\Sigma(\mathcal{S})$.

As we have intimated above our considerations lie entirely in the realm of operator function theory tied to the Hardy space for the line. However the oscillatory integral methods are expected to be effective in multivariable function spaces and for higher rank settings in noncommutative harmonic analysis. This should lead to the identification 
of other closed subspace manifolds with interesting topology and geometry.

Acknowledgements. We wish to thank Alexandru Aleman, Gordon Blower, Jean Esterle, Aristides Katavolos, Alfonso Montes Rodriguez and Donald Sarason for their interest and communications concerning strange limits. The presentation of this paper has benefited considerably from the comments of the anonymous referee, for which we thank him.

The first named author was supported by Engineering and Physical Sciences Research Council grant EP/D050677/1 during parts of the preparation of this paper.

\section{Subspace MANifOldS}

In this section we give some definitions and examples.

Let $\mathcal{H}$ be a separable Hilbert $\operatorname{space}, \operatorname{Proj}(\mathcal{H})$ the set of self-adjoint projections and $\operatorname{Unit}(\mathcal{H})$ the set of unitary operators. We shall routinely identify a closed subspace $\mathcal{K}$ with its associated orthogonal projection, denoted $[\mathcal{K}]$. For $U \in \operatorname{Unit}(\mathcal{H})$ and $T \in \mathcal{B}(\mathcal{H})$ we will write $(\operatorname{Ad} U) T=U T U^{*}$, so that, in particular, $\operatorname{Ad} U[\mathcal{K}]=[U \mathcal{K}]$.

Definition 2.1. (i) A topological subspace manifold in $\mathcal{B}(\mathcal{H})$ of dimension $n$ is a set $\mathcal{M} \subseteq \operatorname{Proj}(\mathcal{H})$, considered with the relative strong operator topology, which is locally homeomorphic to $\mathbb{R}^{n}$.

(ii) A $C^{\infty}$ projection manifold in $\mathcal{B}(\mathcal{H})$ (or $C^{\infty}$ subspace manifold) is a topological subspace manifold $\mathcal{M}$ of dimension $n$ together with an atlas of charts $x_{i}: \mathbb{R}^{n} \rightarrow \mathcal{M}$ (with open domains and ranges covering $\mathcal{M}$ ) for which the coordinate functions $x_{i}^{-1} x_{j}$ (with nonempty domain) are $C^{\infty}$ and such that for each chart $x$ with domain $U_{x}$ there is a dense subspace $\mathcal{D}_{x}$ of $C^{\infty}$ vectors in $\mathcal{H}$; that is, for $f, g \in \mathcal{D}_{x}$, the function $\lambda \mapsto\langle x(\lambda) f, g\rangle$ is $C^{\infty}$ on $U_{x}$.

In fact we bypass the technicalities of (ii) in Definition 2.1 in view of the fact that the smooth subspace manifolds we consider have a stronger locally unitary structure as in the following formal definition.

Definition 2.2. A locally unitary subspace manifold of dimension $n$ in $\mathcal{B}(\mathcal{H})$ is a topological subspace manifold $\mathcal{M}$ such that for each $P$ in $\mathcal{M}$ there is a strong operator topology neighbourhood in $\mathcal{M}$ of the form

$$
\mathcal{N}_{P}=\left\{\left[\rho_{P}(\lambda) P \mathcal{H}\right]: \lambda \in \mathbb{B}_{n}\right\}
$$

where $\rho_{P}: \mathbb{R}^{n} \rightarrow \operatorname{Unit}(\mathcal{H})$ is a strong operator topology continuous representation which is a homeomorphism of $\mathcal{N}_{p} \operatorname{into} \operatorname{Unit}(\mathcal{H})$.

It is well-known that such a representation $\rho_{P}$ does possess a dense subspace of $C^{\infty}$ vectors; see [18], for example. 
We shall consider Beurling subspace manifolds $\mathcal{M}$, given formally in the next definition, together with their complement completions, by which we mean the strong operator topology closure of $\mathcal{M} \cup \mathcal{M}^{\perp}$.

Definition 2.3. A Beurling subspace manifold of dimension $n$ is a topological subspace manifold $\mathcal{M} \subseteq \operatorname{Proj}\left(L^{2}(\mathbb{R})\right)$ of the form

$$
\mathcal{M}=\left\{\left[u_{\lambda} H^{2}(\mathbb{R})\right]: \lambda \in \mathbb{R}^{n}\right\}
$$

where $\lambda \mapsto u_{\lambda}$ is a weak star continuous representation of $\mathbb{R}^{n}$ as unimodular functions, so that $\mathcal{M}$ is locally homeomorphic to $\mathbb{R}^{n}$ by the single chart $x(\lambda)=\left[u_{\lambda} H^{2}(\mathbb{R})\right]$.

If $\psi$ is a non-constant real continuous function on the line then the projections $\left[\exp (i \lambda \psi) H^{2}(\mathbb{R})\right]$, for $\lambda \in \mathbb{R}$, give a one dimensional topological manifold. When $\psi(x)=x$ the closure in $\operatorname{Proj}\left(L^{2}(\mathbb{R})\right)$ adds the subspaces $\{0\}$ and $L^{2}(\mathbb{R})$ and the complement completion, $\Sigma_{\mathrm{a}}$ say, is topologically a circle. On the other hand for $\psi(x)=x^{2}$ we shall see that the closure of $\mathcal{M}\left(\left\{\lambda x^{2}: \lambda \in \mathbb{R}\right\}\right)$ adds $\left[L^{2}\left(\mathbb{R}_{+}\right)\right]$and $\left[L^{2}\left(\mathbb{R}_{-}\right)\right]$ and that the complement completion $\Sigma_{\mathrm{e}}$ is a locally unitary $C^{\infty}$ subspace manifold diffeomorphic to the circle. We see later in Example 4.9 that $\Sigma_{\mathrm{e}}$ and $\Sigma_{\mathrm{a}}$ are, respectively, the equator and a great circle of the Fourier-Plancherel sphere.

The subspaces $K_{\lambda, s}=e^{i \lambda x} e^{i s x^{2}} H^{2}(\mathbb{R})$, for $s<0$ and $\lambda \in \mathbb{R}$, form a subspace manifold $\mathcal{M}$ which arises in the analysis of the invariant subspaces for the Weyl semigroup

$$
\mathcal{W}=\left\{\alpha M_{e^{i \lambda x}} D_{\mu}: \lambda, \mu \geq 0,|\alpha|=1\right\},
$$

where $D_{\mu}$ is the translation unitary $D_{\mu} f(x)=f(x-\mu)$. Indeed it was shown in [7] that the invariant subspaces of $\mathcal{W}$ are the spaces $K_{\lambda, s}$ for $s \leq 0$, together with $L^{2}(t, \infty)$ for $t$ in $\mathbb{R} \cup\{ \pm \infty\}$ and, moreover, that the latter subspaces are in the closure of $\mathcal{M}$. Extending the parameter range of $s$ to include $s \geq 0$ and taking the complement completion one obtains the Fourier-Plancherel sphere. The Volterra circle $\Sigma_{\mathrm{v}}$ consists of the subspaces $L^{2}(t, \infty), L^{2}(-\infty, t)$ for $t \in \mathbb{R} \cup\{ \pm \infty\}$ and is unitarily equivalent to the great circle $\Sigma_{\mathrm{a}}$ via the Fourier-Plancherel transform.

Consider now the three dimensional subspace manifold

$$
\mathcal{M}=\left\{\left[|x|^{i s} e^{i \lambda x} e^{i \mu x^{-1}} H^{2}(\mathbb{R})\right]:(s, \lambda, \mu) \in \mathbb{R}^{3}\right\}
$$

which is the Beurling subspace manifold $\mathcal{M}\left(\mathcal{S}_{2}\right)$ for the space

$$
\mathcal{S}_{2}=\left\{s \log |x|+\lambda x+\mu x^{-1}:(s, \lambda, \mu) \in \mathbb{R}^{3}\right\} .
$$

In Section 4 we give a new direct proof that $\overline{\mathcal{M}}$ is homeomorphic to a closed 3-ball and that $\Sigma\left(\mathcal{S}_{2}\right)=\overline{\mathcal{M}} \cup \overline{\mathcal{M}^{\perp}}$ is a topological 3-sphere.

The Beurling subspace manifolds given above might be more precisely specified as Euclidean Beurling subspace manifolds as there are many other interesting $C^{\infty}$ projection manifolds associated with Hardy 
spaces and unimodular functions. We do not develop this here but we note some fundamental examples.

For $n=1,2, \ldots$ let

$$
\mathcal{M}_{n}=\left\{\left[u_{\underline{\lambda}} H^{2}(\mathbb{R})\right]: \underline{\lambda}=\left(\lambda_{1}, \ldots, \lambda_{n}\right) \in \mathbb{H}^{n}\right\}
$$

where $u_{\lambda}(z)$ is a Blaschke factor inner function with zeros, possibly repeated, at points $\lambda_{1}, \ldots, \lambda_{n}$ in the upper half plane $\mathbb{H}$. Then $\mathcal{M}_{n}$ is a $C^{\infty}$ projection manifold in $\mathcal{B}\left(H^{2}(\mathbb{R})\right)$. Also, $\mathcal{M}_{1}$ consists of codimension 1 projections and is locally unitary with respect to a representation of the Möbius group rather than the Euclidean group. The closure of $\mathcal{M}_{1}$ adds one extra projection, namely $\left[H^{2}(\mathbb{R})\right]$, and is a topological subspace manifold homeomorphic to the 2-sphere, realised as the one point compactification of $\mathbb{H}$.

Manifolds analogous to these, with finite or cofinite dimensional spaces, may be defined also for Bergman Hilbert spaces, and, more generally, in the setting of Hermitian holomorphic vector bundles associated with operators in the Cowen-Douglas theory [1]. For example, with weighted Bergman Hilbert spaces in place of $H^{2}(\mathbb{R})$ one obtains projection manifold realisations of the unit disc with one point compactification closures. That these are unitarily inequivalent was essentially shown in [1] by the construction of curvature invariants for Hermitian holomorphic vector bundles. This underlines the fact that unitary equivalence here is a strong form of geometric equivalence for subspace manifolds. We note the following alternative curvature free approach to this and in subsequent sections we find, similarly, that we do not need to consider curvature. However, it would of course be interesting and useful to define curvature invariants for general projection manifolds.

Let $\alpha \geq 0$ and let $A_{\alpha}^{2}$ be the weighted Bergman Hilbert space of holomorphic functions in the unit disc that are square integrable with respect to $\left(1-|z|^{2}\right)^{\alpha} d A$, where $d A$ is area measure. For $\lambda$ in $\mathbb{D}$ let $u_{\lambda}(z)$ be the inner function $(\lambda-z) /(1-\bar{\lambda} z)$ and let $\mathcal{M}^{\alpha}$ be the projection manifold

$$
\mathcal{M}^{\alpha}=\left\{\left[u_{\lambda} A_{\alpha}^{2}\right]: \lambda \in \mathbb{D}\right\} .
$$

The range of the complementary projection $\left[u_{\lambda} A_{\alpha}^{2}\right]^{\perp}$ is one-dimensional and is spanned by the function $(1-\bar{\lambda} z)^{2-\alpha}$. (See Zhu [21], for example.) A standard argument with eigenvectors (see Theorem 3.6 of Thomson [19] for example) shows that an operator which leaves invariant all these subspaces is necessarily multiplication by the complex conjugate of an $H^{\infty}$ function. Thus if $U$ is a unitary with $\operatorname{Ad} U\left(\mathcal{M}^{\alpha}\right)=\mathcal{M}^{\beta}$ then we may deduce that $\operatorname{Ad} U$ gives an isomorphism between the respective $H^{\infty}$ multiplication algebras. Composing with a unitary automorphism of $\mathcal{B}\left(A_{\beta}^{2}\right)$ which effects the inverse Möbius automorphism on the range algebra we thus obtain a unitary operator $W$ between the Bergman spaces which intertwines multiplication: $M_{z} W=W M_{z}$. 
Thus if $W 1=g$ then $W z^{m}=z^{m} g$. For $\alpha \neq \beta$ this is contrary to $W$ being isometric.

Proposition 2.4. For $\alpha, \beta \geq 0$ the projection manifolds $\mathcal{M}^{\alpha}$ and $\mathcal{M}^{\beta}$ are unitarily equivalent if and only if $\alpha=\beta$.

\section{Strange Limits}

We now develop methods that will be useful for identifying the closures of various Beurling subspace manifolds.

If $\varphi \in L^{\infty}(\mathbb{R})$ then we write $M_{\varphi}$ for the corresponding multiplication operator on $L^{2}(\mathbb{R})$. In particular, if $B$ is a measurable subset of $\mathbb{R}$ and $\chi_{B}$ denotes its characteristic function then $M_{\chi_{B}}$ is the projection $\left[L^{2}(B)\right]$.

Proposition 3.1. Let $B$ be an open subset of $\mathbb{R}$. A sequence of projections $P_{n}$ converges in the strong operator topology to $M_{\chi_{B}}$ if and only if

(i) $\left\|P_{n} \chi_{E}\right\|_{2} \rightarrow\left\|\chi_{E}\right\|_{2}$ for every compact interval $E \subseteq B$, and

(ii) $\left\|P_{n} \chi_{F}\right\|_{2} \rightarrow 0$ for every compact interval $F \subseteq \mathbb{R} \backslash B$.

Proof. Necessity is clear. Suppose that (i) and (ii) hold, and let $E$ and $F$ be disjoint compact intervals. If either is a subset of $\mathbb{R} \backslash B$ then $\left|\left\langle P_{n} \chi_{E}, \chi_{F}\right\rangle\right| \leq\left\|P_{n} \chi_{E}\right\|\left\|P_{n} \chi_{F}\right\| \rightarrow 0$ as $n \rightarrow \infty$. On the other hand, if $E, F \subseteq B$ then for $k \in\{0,1,2,3\}$,

$$
\begin{aligned}
2 \operatorname{Re} i^{k}\left\langle P_{n} \chi_{E}, \chi_{F}\right\rangle & =\left\|P_{n}\left(i^{k} \chi_{E}+\chi_{F}\right)\right\|^{2}-\left\|P_{n} \chi_{E}\right\|^{2}-\left\|P_{n} \chi_{F}\right\|^{2} \\
& \leq\left\|\chi_{E}\right\|^{2}-\left\|P_{n} \chi_{E}\right\|^{2}+\left\|\chi_{F}\right\|^{2}-\left\|P_{n} \chi_{F}\right\|^{2} \rightarrow 0
\end{aligned}
$$

as $n \rightarrow \infty$, and we again conclude that $\left\langle P_{n} \chi_{E}, \chi_{F}\right\rangle \rightarrow 0$ as $n \rightarrow \infty$.

Since the unit ball of $\mathcal{B}\left(L^{2}(\mathbb{R})\right)$ is compact and metrisable in the weak operator topology, there is a positive contraction $C$ which is a weak cluster point of $\left\{P_{n}\right\}$, say $P_{n_{k}} \rightarrow C$ weakly. Now $\left\langle C \chi_{E}, \chi_{F}\right\rangle=0$ for disjoint compact intervals $E, F$ which are each contained in either $B$ or its complement; an approximation argument shows that this remains the case whenever $E$ and $F$ are disjoint bounded measurable sets. Approximation by simple functions yields $\left\langle C \chi_{E}, \chi_{F} f\right\rangle=0$ for every $f \in L^{2}(\mathbb{R})$, and similarly $\left\langle C \chi_{E} g, \chi_{F} f\right\rangle=0$ for all $f, g \in L^{2}(\mathbb{R})$. It follows that $C=M_{\varphi}$ for some $\varphi \in L^{\infty}(\mathbb{R})$ with $0 \leq \varphi \leq 1$ and $\operatorname{supp} \varphi \subseteq B$. If $E$ is a compact interval which is contained in $B$, then $\left\|P_{n_{k}} \chi_{E}\right\|^{2}=\left\langle P_{n_{k}} \chi_{E}, \chi_{E}\right\rangle \rightarrow\left\|\chi_{E}\right\|^{2}=\left\langle\varphi \chi_{E}, \chi_{E}\right\rangle$, which forces $\varphi$ to be equal to 1 almost everywhere on $E$. Since $B$ is open, $C=M_{\chi_{B}}$. Thus the weak limit of $\left\{P_{n_{k}}\right\}$ is a projection, $M_{\chi_{B}}$, from which it follows that SOT- $\lim _{k \rightarrow \infty} P_{n_{k}}=M_{\chi_{B}}$ as well.

Thus every subsequence of $\left\{P_{n}\right\}$ has a subsubsequence whose limit in the strong operator topology is $M_{\chi_{B}}$. Since the unit ball of $\mathcal{B}\left(L^{2}(\mathbb{R})\right)$ is metrisable in this topology, this shows that $P_{n} \rightarrow M_{\chi_{B}}$ strongly. 
We now determine conditions under which we can identify the strong operator topology convergence $P_{n} \rightarrow M_{\chi_{B}}$ for a sequence of Beurling projections $P_{n}=\left[e^{i k_{n} \psi} H^{2}(\mathbb{R})\right]$ as $k_{n} \rightarrow \infty$, where $B$ is a subset of $\mathbb{R}$ determined by $\psi^{\prime}$.

Definition 3.2. A partial function $\psi: \mathbb{R} \rightarrow \mathbb{R}$ is admissible if the following subsets of $\mathbb{R}$ are discrete (that is, they have no accumulation points):

(i) the set $\Gamma(\psi)$ of points at which $\psi$ is undefined, or at which $\psi$ fails to be twice continuously differentiable;

(ii) $\left(\psi^{\prime}\right)^{-1}(0)$; and

(iii) the set $\Lambda(\psi)$ consisting of the points at which $\operatorname{sgn}\left(\psi^{\prime \prime}\right)$ is not locally constant.

For example, non-constant rational functions and the trigonometric functions are easily seen to be admissible, as is the map $x \mapsto \log |x|$.

Recall that $F: L^{2}(\mathbb{R}) \rightarrow L^{2}(\mathbb{R})$ is the unitary Fourier-Plancherel transform. The Hardy space $H^{2}(\mathbb{R})$ is equal to $F^{*} L^{2}(0, \infty)$, so

$$
\left[e^{i k \psi} H^{2}(\mathbb{R})\right]=\operatorname{Ad}\left(M_{e^{i k \psi}} F^{*}\right) M_{\chi_{(0, \infty)}} .
$$

Here, the multiplication operator $M_{e^{i k \psi}}$ is unitary since $e^{i k \psi}$ is unimodular. In particular, if $P=\left[e^{i k \psi} H^{2}(\mathbb{R})\right]$ then

$$
\begin{aligned}
\left\|P \chi_{S}\right\|_{2} & =\left\|\left(\operatorname{Ad}\left(M_{e^{i k \psi}} F^{*}\right) M_{\chi_{(0, \infty)}}\right) \chi_{S}\right\|_{2} \\
& =\left\|M_{e^{i k \psi}} F^{*}\left(M_{\chi_{(0, \infty)}} F M_{e^{i k \psi}}^{*} \chi_{S}\right)\right\|_{2}=\left\|\chi_{(0, \infty)} F\left(e^{-i k \psi} \chi_{S}\right)\right\|_{2} .
\end{aligned}
$$

It is therefore of interest to find an estimate for the Fourier-Plancherel transform $F\left(e^{-i k \psi} \chi_{S}\right)$ on the positive half-line. This is done in the next lemma for admissible functions $\psi$, using an integration by parts in the spirit of [17], Chapter VIII.

Lemma 3.3. Let $\psi$ be admissible and let $S \subseteq \mathbb{R}$ be a compact interval with non-empty interior $S^{\circ}$ such that $\Gamma(\psi) \cap \bar{S}=\emptyset$ and $\psi^{\prime}(S) \subseteq(0, \infty)$. Let $\Delta$ be the function $\Delta(z)=2(z+\alpha)^{-1}$ where $\alpha=\min \left\{\psi^{\prime}(x): x \in S\right\}$ and let $N=\left|\Lambda(\psi) \cap S^{\circ}\right|+2$. Then for $k>0$ and almost every $z>0$, $\sqrt{2 \pi}\left|F\left(e^{-i k \psi} \chi_{S}\right)(k z)\right| \leq N k^{-1} \Delta(z)$.

Proof. Since $\Lambda(\psi)$ is discrete, finitely many points in $\Lambda(\psi)$ lie in the interior of $S$, say $\lambda_{2}<\lambda_{3}<\cdots<\lambda_{N-1}$. We also write $\lambda_{1}, \lambda_{N}$ for the boundary points of $S$ so that $S=\left[\lambda_{1}, \lambda_{N}\right]$. Since $\Gamma(\psi) \cap S=\emptyset$, the function $\psi$ is twice continuously differentiable on $S$ and for $z>0$,

$$
\begin{aligned}
\sqrt{2 \pi}\left|F\left(e^{-i k \psi} \chi_{S}\right)(k z)\right|=\left|\int_{S} e^{-i k(x z+\psi(x))} d x\right| \\
=\left|\int_{S} \frac{i}{k\left(z+\psi^{\prime}(x)\right)} \frac{d}{d x}\left(e^{-i k(x z+\psi(x))}\right) d x\right|
\end{aligned}
$$




$$
\begin{aligned}
& =\frac{1}{k}\left|\left[\frac{e^{-i k(x z+\psi(x))}}{z+\psi^{\prime}(x)}\right]_{\lambda_{1}}^{\lambda_{N}}-\int_{S} \frac{d}{d x}\left(\frac{1}{z+\psi^{\prime}(x)}\right) e^{-i k(x z+\psi(x))} d x\right| \\
& \leq \frac{1}{k}\left(\Delta(z)+\int_{S}\left|\frac{d}{d x}\left(\frac{1}{z+\psi^{\prime}(x)}\right)\right| d x\right) .
\end{aligned}
$$

Let $j \in\{1,2, \ldots, N-1\}$. For $x \in\left(\lambda_{j}, \lambda_{j+1}\right)$, the quantity

$$
\operatorname{sgn} \frac{d}{d x}\left(\frac{1}{z+\psi^{\prime}(x)}\right)=\operatorname{sgn} \frac{-\psi^{\prime \prime}(x)}{\left(z+\psi^{\prime}(x)\right)^{2}}=-\operatorname{sgn}\left(\psi^{\prime \prime}(x)\right)
$$

is constant, say $\sigma_{j} \in\{-1,0,1\}$. So

$$
\begin{aligned}
\int_{S}\left|\frac{d}{d x}\left(\frac{1}{z+\psi^{\prime}(x)}\right)\right| d x & =\sum_{j=1}^{N-1} \sigma_{j} \int_{\lambda_{j}}^{\lambda_{j+1}} \frac{d}{d x}\left(\frac{1}{z+\psi^{\prime}(x)}\right) d x \\
& =\sum_{j=1}^{N-1} \sigma_{j}\left[\frac{1}{z+\psi^{\prime}(x)}\right]_{\lambda_{j}}^{\lambda_{j+1}} \\
& \leq(N-1) \Delta(z) .
\end{aligned}
$$

The result follows.

Theorem 3.4. Let $k_{n}$ be a sequence of positive numbers with $k_{n} \rightarrow \infty$ as $n \rightarrow \infty$, let $\psi$ be admissible and let $P_{n}=\left[e^{i k_{n} \psi} H^{2}(\mathbb{R})\right]$ for $n \in \mathbb{N}$. Then $P_{n} \stackrel{\text { SOT }}{\rightarrow}\left[L^{2}\left(B_{-}\right)\right]$as $n \rightarrow \infty$, where $B_{-}=\left(\psi^{\prime}\right)^{-1}((-\infty, 0))$.

Proof. Let $B_{+}=\left(\psi^{\prime}\right)^{-1}((0, \infty))$ and $B_{0}=\left(\psi^{\prime}\right)^{-1}(0)$. Let $S$ be a compact subinterval of $B_{+}$of positive length which does not intersect $\Gamma(\psi)$. We will show that $P_{n} \chi_{S} \rightarrow 0$. Since $P_{n}=\operatorname{Ad}\left(M_{e^{i k_{n} \psi}} F^{*}\right) M_{\chi_{(0, \infty)}}$,

$$
\begin{aligned}
\left\|P_{n} \chi_{S}\right\|_{2}^{2} & =\left\|\chi_{(0, \infty)} F\left(e^{-i k_{n} \psi} \chi_{S}\right)\right\|_{2}^{2} \\
& =\int_{0}^{\infty}\left|F\left(e^{-i k_{n} \psi} \chi_{S}\right)(y)\right|^{2} d y \\
& =k_{n} \int_{0}^{\infty}\left|F\left(e^{-i k_{n} \psi} \chi_{S}\right)\left(k_{n} z\right)\right|^{2} d z
\end{aligned}
$$

where we have made the change of variables $y=k_{n} z$. We apply Lemma 3.3:

$$
\left\|P_{n} \chi_{S}\right\|_{2}^{2} \leq \frac{k_{n}}{2 \pi} \int_{0}^{\infty}\left(\frac{N}{k_{n}}\right)^{2}|\Delta(z)|^{2} d z=\frac{N^{2}}{2 \pi k_{n}}\left\|\chi_{(0, \infty)} \Delta\right\|_{2}^{2} \rightarrow 0
$$

as $n \rightarrow \infty$, where $\Delta(z) \in L^{2}\left(\chi_{(0, \infty)} d z\right)$ and $N \in \mathbb{N}$ are defined as in Lemma 3.3.

So $P_{n} \chi_{S} \rightarrow 0$ whenever $S$ is a compact subinterval of $B_{+} \backslash \Gamma(\psi)$. By the discreteness of $B_{0} \cup \Gamma(\psi)$, the same is true if $S$ is a compact subinterval of $\mathbb{R} \backslash B=B_{+} \cup\left(B_{0} \cup \Gamma(\psi)\right)$ where $B=B_{-} \backslash \Gamma(\psi)$. Since $\psi$ is continuously differentiable on $\mathbb{R} \backslash \Gamma(\psi)$ it follows that $B$ is open.

Let $\varphi=-\psi$, let $Q_{n}=\left[e^{i k_{n} \varphi} H^{2}(\mathbb{R})\right]$ and let $C$ be the conjugation operator $C f=\bar{f}$ for $f \in L^{2}(\mathbb{R})$. Since $C H^{2}(\mathbb{R})=\overline{H^{2}(\mathbb{R})}=H^{2}(\mathbb{R})^{\perp}$, it 
follows that $C P_{n}^{\perp} C$ is a self-adjoint projection whose range is $Q_{n} L^{2}(\mathbb{R})$, so $Q_{n}=C P_{n}^{\perp} C$. Applying the argument above to $\varphi$ instead of $\psi$ shows that $Q_{n} \chi_{T} \rightarrow 0$ whenever $T$ is a compact subinterval of $B$. Hence $P_{n} \chi_{T} \rightarrow \chi_{T}$.

By Proposition 3.1, $P_{n} \stackrel{\text { SOT }}{\rightarrow} M_{\chi_{B}}=M_{\chi_{B_{-}}}=\left[L^{2}\left(B_{-}\right)\right]$as $n \rightarrow \infty$.

The theorem enables us to compute immediately a wide variety of strange limits $P_{n} \stackrel{\text { SOT }}{\longrightarrow} P$, so-called because while every nonzero function in the range of $P_{n}$ has full support, those for $P$ itself are supported in a proper measurable set. For example, $\left[e^{-i n x^{2}} H^{2}(\mathbb{R})\right] \stackrel{\text { SOT }}{\rightarrow}\left[L^{2}\left(\mathbb{R}_{+}\right)\right]$as $n \rightarrow \infty$, and $\left[e^{i n\left(x^{3}+b x^{2}+c x\right)} H^{2}(\mathbb{R})\right] \stackrel{\text { SOT }}{\rightarrow}\left[L^{2}([\alpha, \beta])\right]$ if the roots $\{\alpha, \beta\}$ of the equation $3 x^{2}+2 b x+c=0$ are real, and has limit 0 otherwise. We also remark that when $\psi$ is admissible,

$$
\underset{n \rightarrow \infty}{\operatorname{SOT}-\lim }\left[e^{i n \psi} H^{2}(\mathbb{R})\right]=\left(\underset{n \rightarrow \infty}{\operatorname{SOT}-\lim }\left[e^{-i n \psi} H^{2}(\mathbb{R})\right]\right)^{\perp} .
$$

While admissible functions are adequate for our applications one can perhaps partially relax this constraint. However, we are unaware of a general formula for the limit when $\psi$ is real, measurable and locally bounded.

The next corollary will play a part in the proof of Theorem 4.2.

Corollary 3.5. Let $k_{n}, \psi$ and $B_{ \pm}$be as above, let $\psi_{n}$ be a sequence of admissible functions and let $P_{n}=\left[e^{i k_{n} \psi_{n}} H^{2}(\mathbb{R})\right]$. Suppose that the set $\Gamma=\Gamma(\psi) \cup \bigcup_{n} \Gamma\left(\psi_{n}\right)$ is discrete. Let

$$
\mathcal{I}=\{S \subseteq \mathbb{R} \backslash \Gamma: S=[\alpha, \beta], \alpha<\beta\}
$$

and suppose that the quantity $N(S)=\sup _{n}\left|\Lambda\left(\psi_{n}\right) \cap S^{\circ}\right|$ is finite for every $S \in \mathcal{I}$. If $\psi_{n}^{\prime} \rightarrow \psi^{\prime}$ uniformly on $S$ for every interval $S \in \mathcal{I}$ then $P_{n} \rightarrow\left[L^{2}\left(B_{-}\right)\right]$strongly as $n \rightarrow \infty$.

Proof. Choose a compact subinterval $S \subseteq B_{+} \backslash \Gamma$. Let

$$
\alpha=\min \left\{\psi^{\prime}(x): x \in S\right\} \quad \text { and } \quad \alpha_{n}=\min \left\{\psi_{n}^{\prime}(x): x \in S\right\} \text { for } n \in \mathbb{N} .
$$

Pick $n$ sufficiently large that $\left\|\psi_{n}^{\prime}-\psi^{\prime}\right\|_{S}<\alpha / 2$; then $\alpha_{n}>\alpha / 2>0$. Writing

$$
\Delta_{n}(z)=2\left(z+\alpha_{n}\right)^{-1} \text { and } \tilde{\Delta}(z):=2(z+\alpha / 2)^{-1},
$$

Lemma 3.3 applies as before to show that

$$
\left\|P_{n} \chi_{S}\right\|^{2} \leq \frac{N(S)^{2}}{2 \pi k_{n}}\left\|\chi_{(0, \infty)} \Delta_{n}\right\|_{2}^{2} \leq \frac{N(S)^{2}}{2 \pi k_{n}}\left\|\chi_{(0, \infty)} \tilde{\Delta}\right\|_{2}^{2}
$$

Since $\tilde{\Delta}(z) \in L^{2}\left(\chi_{(0, \infty)} d z\right)$, this shows that $P_{n} \chi_{S} \rightarrow 0$. The remainder of the proof proceeds as in Theorem 3.4.

Beurling's characterisation of invariant subspaces for the bilateral shift operator when transferred to the setting $L^{2}(\mathbb{R})$ amounts to the 
identification of $\operatorname{Lat}\left\{M_{e^{i \lambda x}}: \lambda \geq 0\right\}$ with the disjoint union

$$
\begin{aligned}
& \left\{u H^{2}(\mathbb{R}): u \text { unimodular }\right\} \cup\left\{L^{2}(E): E \text { measurable }\right\} \\
& \quad=\mathcal{M}_{\text {pure }} \cup \operatorname{Lat}\left\{M_{\varphi}: \varphi \in L^{\infty}(\mathbb{R})\right\} .
\end{aligned}
$$

Here Lat $\mathcal{A}$ denotes the lattice of closed invariant subspaces for a family of operators $\mathcal{A}$, and $\mathcal{M}_{\text {pure }}$ is the set of invariant subspaces $K$ which are purely invariant in the sense that the intersection of the subspaces $M_{e^{i \lambda x}} K$ for $\lambda \geq 0$ is trivial. We now use the methods of this section to show that Lat $\left(L^{\infty}(\mathbb{R})\right) \subseteq \overline{\mathcal{M}_{\text {pure }}}$. This seems to be a previously unobserved feature in the classical setting which may well have a wider manifestation. However, the authors are unaware of any general results of this nature.

Lemma 3.6. Let $m$ denote Lebesgue measure on $\mathbb{R}$. If $B \subseteq \mathbb{R}$ is measurable and $\varepsilon>0$ then there is a countable disjoint union of open intervals $V$ such that $\partial V$ is discrete and $m(V \triangle B)<\varepsilon$.

Proof. Fix $n \in \mathbb{Z}$ and write $B_{n}=B \cap(n, n+1)$ and $\varepsilon_{n}=2^{-|n|} \varepsilon / 3$. Using elementary properties of Lebesgue measure, we can find a set $U_{n}=\bigcup_{j>1} I_{j} \supseteq B_{n}$ such that $\left\{I_{j}\right\}_{j \geq 1}$ are disjoint open subintervals of $(n, n+1)$ and $m\left(U_{n} \backslash B_{n}\right)<\frac{1}{2} \varepsilon_{n}$. Pick $k$ so that $\sum_{j>k} m\left(I_{j}\right)<\frac{1}{2} \varepsilon_{n}$ and let $V_{n}=\bigcup_{1 \leq j \leq k} I_{j}$. Now

$$
V_{n} \triangle B_{n}=\left(\left(U_{n} \backslash B_{n}\right) \cap V_{n}\right) \cup\left(B_{n} \backslash V_{n}\right) \subseteq U_{n} \backslash B_{n} \cup \bigcup_{j>k} I_{j},
$$

so $m\left(V_{n} \triangle B_{n}\right)<\frac{1}{2} \varepsilon_{n}+\frac{1}{2} \varepsilon_{n}=\varepsilon_{n}$.

Repeat for each $n \in \mathbb{Z}$ and let $V=\bigcup_{n \in \mathbb{Z}} V_{n}$. Then

$$
m(V \triangle B)=\sum_{n \in \mathbb{Z}} m\left(V_{n} \triangle B_{n}\right)<\sum_{n \in \mathbb{Z}} \varepsilon_{n}=\varepsilon
$$

and $\partial V$ is discrete, since $\partial V \cap[n, n+1]$ is finite for each $n$.

Theorem 3.7. If $B$ is any measurable subset of $\mathbb{R}$ then there is a sequence of projections $P_{n}=\left[e^{i k_{n} \psi_{n}} H^{2}(\mathbb{R})\right]$ where $k_{n}>0$ and each $\psi_{n}$ is a real-valued function such that SOT- $\lim _{n \rightarrow \infty} P_{n}=M_{\chi_{B}}$.

Proof. Let $\varepsilon>0$. By Lemma 3.6, we can find a countable disjoint union of open intervals $V_{\varepsilon}$ such that $\partial V_{\varepsilon}$ is discrete and $m\left(V_{\varepsilon} \triangle B\right)<\varepsilon$. The function $\psi_{\varepsilon}(x)=x\left(\chi_{\mathbb{R} \backslash V_{\varepsilon}}-\chi_{V_{\varepsilon}}\right)$ satisfies $\Gamma\left(\psi_{\varepsilon}\right) \cup \Lambda\left(\psi_{\varepsilon}\right) \subseteq \partial V_{\varepsilon}$ and $\left(\psi_{\varepsilon}^{\prime}\right)^{-1}(0)=\emptyset$, so $\psi_{\varepsilon}$ is admissible. Let $P_{\varepsilon, n}=\left[e^{i n \psi_{\varepsilon}} H^{2}(\mathbb{R})\right]$. By Theorem 3.4, sot- $\lim _{n \rightarrow \infty} P_{\varepsilon, n}=M_{\chi_{V_{\varepsilon}}}$, and we also have $M_{\chi_{V_{\varepsilon}}} \rightarrow M_{\chi_{B}}$ strongly as $\varepsilon \rightarrow 0$.

Let $d$ be a metric inducing the strong operator topology on the unit ball of $\mathcal{B}\left(L^{2}(\mathbb{R})\right)$ and let $n \in \mathbb{N}$. Choose $\varepsilon_{n}>0$ such that $d\left(M_{\chi_{V_{\varepsilon}}}, M_{\chi_{B}}\right)<1 / 2 n$ and then choose $k \in \mathbb{N}$ so that $P_{n}:=P_{\varepsilon_{n}, k}$ satisfies $d\left(P_{n}, M_{\chi_{V_{\varepsilon_{n}}}}\right)<1 / 2 n$. Now $d\left(P_{n}, M_{\chi_{B}}\right)<1 / n$ so $P_{n} \rightarrow M_{\chi_{B}}$ strongly as $n \rightarrow \infty$. 


\section{Closures of Beurling subspace manifolds}

We now obtain sufficient conditions under which Beurling subspace manifolds $\mathcal{M}(\mathcal{S})$ have closures, in the strong operator topology, which are compact. Using this we construct various $n$-spheres and $n$-balls in $\operatorname{Proj}\left(L^{2}(\mathbb{R})\right)$. At the end of the section we pose some further lines of enquiry.

Let $f=\left(f_{1}, f_{2}, \ldots, f_{n}\right)$ be an $n$-tuple of functions $f_{j}: \mathbb{R} \rightarrow \mathbb{R}$. We write $\langle f, \lambda\rangle=\lambda_{1} f_{1}+\lambda_{2} f_{2}+\cdots+\lambda_{n} f_{n}$ for $\lambda \in \mathbb{R}^{n}$, and

$$
\mathcal{S}_{f}=\left\{\langle f, \lambda\rangle: \lambda \in \mathbb{R}^{n}\right\} .
$$

Definition 4.1. The $n$-tuple $f$ is admissible if

(i) the set $\left\{f_{1}, f_{2}, \ldots, f_{n}\right\}$ is linearly independent over $\mathbb{R}$;

(ii) every nonzero function in $\mathcal{S}_{f}$ is admissible; and

(iii) $\sup _{g \in \mathcal{S}_{f} \backslash\{0\}}|K \cap \Lambda(g)|<\infty$ for each compact set $K \subseteq \mathbb{R}$.

We will also write $\Gamma(f)=\bigcup_{j=1}^{n} \Gamma\left(f_{j}\right)$ and remark that this is equal to $\bigcup_{g \in \mathcal{S}_{f}} \Gamma(g)$ and is plainly discrete.

Given an admissible $n$-tuple $f$, let $\theta: \mathbb{R}^{n} \rightarrow \operatorname{Proj}\left(L^{2}(\mathbb{R})\right)$ be the map $\lambda \mapsto\left[e^{i\langle f, \lambda\rangle} H^{2}(\mathbb{R})\right]$. Observe that $\theta$ is strongly continuous, since if $\lambda^{(k)} \rightarrow \lambda$ in $\mathbb{R}^{n}$ then $\left\langle f, \lambda^{(k)}\right\rangle \rightarrow\langle f, \lambda\rangle$ uniformly on compact subsets of $\mathbb{R} \backslash \Gamma(f)$ and so

$$
\theta\left(\lambda^{(k)}\right)=\operatorname{Ad}\left(M_{\left.e^{i\langle f, \lambda}(k)\right\rangle}\right)\left[H^{2}(\mathbb{R})\right] \stackrel{\text { SOT }}{\rightarrow} \operatorname{Ad}\left(M_{e^{i\langle f, \lambda\rangle}}\right)\left[H^{2}(\mathbb{R})\right]=\theta(\lambda)
$$

as $k \rightarrow \infty$.

We write $\mathcal{M}\left(\mathcal{S}_{f}\right)=\theta\left(\mathbb{R}^{n}\right)$. We will shortly see that $\mathcal{M}\left(\mathcal{S}_{f}\right)$ is a Beurling subspace manifold.

Theorem 4.2. Given an admissible n-tuple $f$, the closure of the range of $\theta$ in the strong operator topology is

$$
\overline{\mathcal{M}\left(\mathcal{S}_{f}\right)}=\theta\left(\mathbb{R}^{n}\right) \cup\left\{\left[L^{2}\left(\left(\psi^{\prime}\right)^{-1}((-\infty, 0))\right)\right]: \psi \in \mathcal{S}_{f} \backslash\{0\}\right\} .
$$

Proof. Let $\lambda^{(k)}$ be a sequence in $\mathbb{R}^{n}$ and let $P_{k}=\theta\left(\lambda^{(k)}\right)$ be the corresponding sequence of projections. Passing to a subsequence, we may assume that $\lambda^{(k)}$ converges to a vector $\lambda \in(\mathbb{R} \cup\{ \pm \infty\})^{n}$ as $k \rightarrow \infty$. If $\lambda$ actually lies in $\mathbb{R}^{n}$ then $P_{k} \rightarrow \theta(\lambda)$ by the continuity of $\theta$. Otherwise, if $\alpha_{k}=\max _{j}\left|\lambda_{j}^{(k)}\right|$ then $\alpha_{k} \rightarrow \infty$ as $k \rightarrow \infty$. Let $\mu^{(k)}=\alpha_{k}^{-1} \lambda^{(k)}$. Passing to a subsequence, we may assume that $\alpha_{k}=\left|\lambda_{j_{0}}^{(k)}\right|$ for some $j_{0}$ independent of $k$, and that $\mu^{(k)} \rightarrow \mu$ for some $\mu \in[-1,1]^{n}$. Since $\mu_{j_{0}}= \pm 1$, this limit $\mu$ is nonzero. Now $P_{k}=\left[e^{i \alpha_{k} \psi_{k}} H^{2}(\mathbb{R})\right]$ where $\psi_{k}=\left\langle f_{k}, \mu^{(k)}\right\rangle$, and if $\psi=\langle f, \mu\rangle$ then $\psi_{k}^{\prime} \rightarrow \psi^{\prime}$ uniformly on compact subsets of $\mathbb{R} \backslash \Gamma(f)$. By Corollary 3.5, $P_{k} \rightarrow\left[L^{2}\left(\left(\psi^{\prime}\right)^{-1}((-\infty, 0))\right)\right]$.

Conversely, if $\psi \in \mathcal{S}_{f} \backslash\{0\}$ then by Theorem 3.4,

$$
\left[L^{2}\left(\left(\psi^{\prime}\right)^{-1}((-\infty, 0))\right)\right]=\underset{n \rightarrow \infty}{\operatorname{SOT}-\lim }\left[e^{i n \psi} H^{2}(\mathbb{R})\right] \in \overline{\mathcal{M}\left(\mathcal{S}_{f}\right)} .
$$


Definition 4.3. Given an admissible $n$-tuple $f$, let $\sim$ be the equivalence relation defined on $\overline{\mathbb{B}^{n}}$ by $\lambda \sim \mu$ if $\lambda=\mu$ or

$\lambda, \mu \in S^{n-1}$ and $m\left(\left\{x:\left\langle f^{\prime}, \lambda\right\rangle(x)>0\right\} \triangle\left\{x:\left\langle f^{\prime}, \mu\right\rangle(x)>0\right\}\right)=0$.

Here $f^{\prime}=\left(f_{1}^{\prime}, \ldots, f_{n}^{\prime}\right)$ and $m$ is Lebesgue measure on $\mathbb{R}$. We write $\overline{\mathbb{B}^{n}} / \sim$ for the corresponding topological quotient space.

Proposition 4.4. The topological space $\overline{\mathbb{B}^{n}} / \sim$ is homeomorphic to $\overline{\mathcal{M}}=\overline{\mathcal{M}\left(\mathcal{S}_{f}\right)}$. In particular, $\overline{\mathcal{M}}$ is compact.

Proof. Let $\theta$ be the continuous map $\mathbb{R}^{n} \rightarrow \overline{\mathcal{M}}$ defined above. Observe that $\theta$ is injective: for $\theta(\lambda)=\theta(\mu)$ if and only if $e^{i\langle f, \lambda-\mu\rangle} H^{2}(\mathbb{R})=H^{2}(\mathbb{R})$ which implies that the function $g=\langle f, \lambda-\mu\rangle$ is constant modulo $2 \pi$ almost everywhere, so $g$ cannot be admissible. Since $g \in \mathcal{S}_{f}$, we conclude that $g=0$; by linear independence, $\lambda=\mu$.

Let $\alpha: \mathbb{R}^{n} \rightarrow \mathbb{B}^{n}$ be a homeomorphism of the form

$$
\alpha: \lambda \mapsto \rho(\|\lambda\|) \lambda\|\lambda\|^{-1}
$$

where $\rho:[0, \infty) \rightarrow[0,1)$ is a homeomorphism. Consider the injective continuous map $\varphi=\theta \circ \alpha^{-1}: \mathbb{B}^{n} \rightarrow \overline{\mathcal{M}}$. We extend this to $\overline{\mathbb{B}^{n}}$ by defining $\varphi(\lambda)=\lim _{r \uparrow 1} \varphi(r \lambda)$ for $\lambda \in S^{n-1}$; this limit exists by Theorem 3.4. The extended map is also continuous by Corollary 3.5 and surjective by Theorem 4.2. Since $\varphi(\lambda)=\varphi(\mu)$ if and only if $\lambda \sim \mu$, it follows that $\varphi$ induces a homeomorphism from the compact space $\overline{\mathbb{B}^{n}} / \sim$ onto the Hausdorff space $\overline{\mathcal{M}}$.

Remark 4.5. This proof shows that $\theta: \mathbb{R}^{n} \rightarrow \mathcal{M}\left(\mathcal{S}_{f}\right)$ is a homeomorphism when $f$ is admissible, and so $\mathcal{M}\left(\mathcal{S}_{f}\right)$ is indeed a Beurling subspace manifold.

Determining the precise nature of the quotient space $\overline{\mathbb{B}^{n}} / \sim$ seems difficult in general. However, Proposition 4.8 below shows that there are no surprises when $n=2$.

Lemma 4.6. Let $\mathcal{I}$ be any set of closed, pairwise disjoint intervals, each contained in $(0,1)$. There exists a continuous non-decreasing surjection $\beta:[0,1] \rightarrow[0,1]$ such that $\beta(x)=\beta(y)$ if and only if $x, y \in I$ for some $I \in \mathcal{I}$.

Proof. We may assume without loss of generality that each $I \in \mathcal{I}$ is of the form $I=[a, b]$ with $0<a<b<1$. Observe that $\mathcal{I}$ must be countable since for each $n \geq 1$, the set $\left\{[a, b] \in \mathcal{I}: b-a>n^{-1}\right\}$ is finite. Enumerate $\mathcal{I}$ in decreasing size, so that $\mathcal{I}=\left\{I_{1}, I_{2}, \ldots\right\}$ where $\left|I_{n}\right| \geq\left|I_{n+1}\right|$ for $n \geq 1$. Assign values $a_{n}$ on $I_{n}$ to create a partially defined increasing function $\beta_{1}$ on $\bigcup \mathcal{I}$ as follows. Set $\beta_{1}(0)=0$ and $\beta_{1}(1)=1$. Then set $a_{1}=\frac{1}{2}$ and $a_{2}=\frac{1}{4}$ or $\frac{3}{4}$, according to whether the new interval $a_{2}$ is to the left or the right of $a_{1}$. Continue "interpolating dyadically" in this way, so that $a_{n+1}$ is chosen as the mean of $\beta_{1}(\ell)$ 
and $\beta_{1}(r)$ where $\ell$ (respectively, $r$ ) is the point of $\{0,1\} \cup I_{1} \cup \cdots \cup I_{n}$ immediately to the left (respectively, right) of $I_{n+1}$.

Let $K$ be the closure in $[0,1]$ of $\{0,1\} \cup \bigcup \mathcal{I}$. Let $U$ be the complement of $K$, an open set in $[0,1]$ and thus a union of open intervals, which we call "gaps". We call a gap "good" if it is of the form $J=\left(b, a^{\prime}\right)$ where $I_{n}=[a, b]$ and $I_{m}=\left[a^{\prime}, b^{\prime}\right]$ are intervals in $\mathcal{I}$, and "bad" otherwise. We can extend $\beta_{1}$ to a good gap $J$ by linear interpolation between $a_{n}$ and $a_{m}$. On the other hand, a bad gap $J^{\prime}$ must have at least one of its end points a limit of endpoints of intervals $I \in \mathcal{I}$, so $\beta_{1}$ can be extended to the closure $\overline{J^{\prime}}$ as a non-decreasing function in a unique way, namely by being constant on $\overline{J^{\prime}}$. So we get a continuous increasing function $\beta_{1}$ defined on $[0,1]$. We now correct the constancy on the intervals $\overline{J^{\prime}}$ by forming $\beta_{3}=\beta_{1}+\beta_{2}$ where

$$
\beta_{2}=\sum_{\operatorname{bad} J^{\prime}} \beta_{J^{\prime}}
$$

and $\beta_{J^{\prime}}$ is the continuous map on $[0,1]$ which takes the value 0 to the left of $J^{\prime}$, the value $\left|J^{\prime}\right|$ to the right and is linear on $J^{\prime}$. (None of these $\beta_{J^{\prime}}$ spoil constancy on the $I_{n}$.) Continuity of $\beta_{3}$ is clear. Finally, let $\beta=c \beta_{3}$ with $c=\left(1+\sum_{\text {bad } J^{\prime}}\left|J^{\prime}\right|\right)^{-1}$ so that $\beta(1)=1$.

Lemma 4.7. Let $\mathcal{I}$ be a set of closed, pairwise disjoint proper arcs in $\mathbb{T}$. Then $\overline{\mathbb{D}} / \mathcal{I}$ is homeomorphic to $\overline{\mathbb{D}}$.

Proof. We may assume that $(1,0)$ does not belong to any set in $\mathcal{I}$. Transfer $\mathcal{I}$ to $[0,1]$ in the obvious manner and apply the previous lemma to obtain an increasing continuous surjection $\beta:[0,1] \rightarrow[0,1]$ such that $\beta(x)=\beta(y)$ if and only if $\left\{e^{2 \pi i x}, e^{2 \pi i y}\right\} \subseteq I$ for some $I \in \mathcal{I}$. The $\operatorname{map} \varphi: \overline{\mathbb{D}} \rightarrow \overline{\mathbb{D}}$ given by $\varphi\left(r e^{2 \pi i x}\right)=r e^{2 \pi i(r \beta(x)+(1-r) x)}$ for $r, x \in(0,1]$ and $\varphi(0)=0$ induces a continuous bijection $\overline{\mathbb{D}} / \mathcal{I} \rightarrow \overline{\mathbb{D}}$, which is a homeomorphism since $\overline{\mathbb{D}} / \mathcal{I}$ is compact.

Proposition 4.8. For every admissible pair $(f, g)$, the closure in the strong operator topology of the Beurling subspace manifold $\mathcal{M}\left(\mathcal{S}_{(f, g)}\right)$ is homeomorphic to $\overline{\mathbb{D}}$.

Proof. Let $\sim$ be the equivalence relation on $\overline{\mathbb{D}}$ of Definition 4.3 , with equivalence classes $\{[\lambda]: \lambda \in \overline{\mathbb{D}}\}$. Observe that $\lambda \not-\lambda$ and $\lambda \sim \mu$ if and only if $-\lambda \sim-\mu$. Also, the equivalence classes are connected: if $\lambda \sim \mu$ then the shorter of the two arcs joining $\lambda$ to $\mu$ is contained in $[\lambda]=[\mu]$.

We show that the equivalence classes are also closed. If there is a non-trivial equivalence class then we can make a different choice of $f$ and $g$ without changing the set $\mathcal{S}_{(f, g)}$ to arrange that $(1,0) \sim(0,-1)$, and so also that $(-1,0) \sim(0,1)$. Any remaining equivalence classes are of the form $[\lambda]$ where $\lambda=\left(\lambda_{1}, \lambda_{2}\right)$ with $\lambda_{1} \lambda_{2}>0$; we may assume that $\lambda_{1}>0$ and $\lambda_{2}>0$. Let $h=g^{\prime} / f^{\prime}$ and let $\alpha=-\lambda_{1} / \lambda_{2}$. Then 
$[\lambda]=[\lambda]_{+} \cap[\lambda]_{-}$where

$$
\begin{aligned}
& {[\lambda]_{+}=\left\{\mu:\left\{f^{\prime}>0\right\} \cap\left(\{h>\alpha\} \triangle\left\{h>-\mu_{1} / \mu_{2}\right\}\right) \text { is null }\right\} \text { and }} \\
& {[\lambda]_{-}=\left\{\mu:\left\{f^{\prime}<0\right\} \cap\left(\{h<\alpha\} \triangle\left\{h<-\mu_{1} / \mu_{2}\right\}\right) \text { is null }\right\} .}
\end{aligned}
$$

Here and below we employ abbreviations of the form $\{P(\varphi)\}$ to mean the set $\{x \in \mathbb{R}: P(\varphi(x))\}$ where $\varphi: \mathbb{R} \rightarrow \mathbb{R}$ and $P$ is a predicate depending on a real parameter.

Note that for any constant $k$, the set $\{x: h(x)=k\}$ is null; for if not, then $f^{\prime}-k g^{\prime}$ takes the value 0 on a non-null set, so $f-k g$ is not admissible, whence $f=k g$; but $f$ and $g$ are independent. For $\gamma>0$, let $u_{\gamma}$ be the unit vector $(\gamma, 1) /\left(\gamma^{2}+1\right)^{1 / 2}$. Each $[\lambda]_{+}$is clearly connected. It is also closed, for if $b>a>0$ and $\left\{u_{\gamma}: \gamma \in(a, b)\right\} \subseteq[\lambda]_{+}$ then $\{\alpha<h \leq-b\} \cap\left\{f^{\prime}>0\right\}$ is equal to the union of the null sets $\left\{f^{\prime}>0, h=-b\right\}$ and $\bigcup_{n \geq 1}\left\{\alpha<h \leq-b-n^{-1}\right\} \cap\left\{f^{\prime}>0\right\}$, so is null; hence $u_{b}$, and by a similar argument $u_{a}$, lie in $[\lambda]_{+}$. The class $[\lambda]_{-}$is closed for the same reasons, and hence each class $[\lambda]$ is a closed subarc of $\mathbb{T}$. Now Proposition 4.4 and Lemma 4.7 complete the proof.

Given an admissible $n$-tuple $f$, let $\Sigma\left(\mathcal{S}_{f}\right)$ denote the complement completion of $\mathcal{M}\left(\mathcal{S}_{f}\right)$; that is, the closure of $\mathcal{M}\left(\mathcal{S}_{f}\right) \cup \mathcal{M}\left(\mathcal{S}_{f}\right)^{\perp}$ in the strong operator topology. It is not hard to see that $\mathcal{M}\left(\mathcal{S}_{f}\right)$ and $\mathcal{M}\left(\mathcal{S}_{f}\right)^{\perp}$ are disjoint, and the boundaries of these sets are equal by Theorem 4.2. From this it follows that provided $\overline{\mathcal{M}\left(\mathcal{S}_{f}\right)}$ is homeomorphic to $\overline{\mathbb{B}^{n}}$, the set $\Sigma\left(\mathcal{S}_{f}\right)$ is homeomorphic to $S^{n}$ since it is homeomorphic to the union of two copies of $\overline{\mathbb{B}^{n}}$ joined at their boundaries.

Recall that if $f \in H^{2}(\mathbb{R})$ and $f \neq 0$ then $f^{-1}(0)$ has Lebesgue measure zero (see Theorem 6.13 of [4] and Corollary 6.4 .2 of [13]). This will be used several times below, where we refer to the result as the F. \& M. Riesz theorem.

We also remind the reader that if $\mathcal{H}$ is a Hilbert space then the operations of closed linear span and intersection of closed subspaces impose a natural lattice structure on $\operatorname{Proj}(\mathcal{H})$. The corresponding partial order is simply $\left[\mathcal{K}_{1}\right] \leq\left[\mathcal{K}_{2}\right] \Longleftrightarrow \mathcal{K}_{1} \subseteq \mathcal{K}_{2}$ for closed subspaces $\mathcal{K}_{i} \subseteq \mathcal{H}$. A nest is a chain in $\operatorname{Proj}(\mathcal{H})$ containing 0 and $I$ which is complete with respect to these lattice operations [3]. A nest is said to be continuous if it contains no element with an immediate predecessor in the nest.

Example 4.9. The Fourier-Plancherel sphere is the set of projections

$$
\Sigma_{\mathrm{FP}}=\Sigma\left(\mathcal{S}_{f}\right) \quad \text { where } f \text { is the admissible pair } f=\left(x, x^{2}\right) .
$$

By Proposition 4.8 or the direct arguments of [7], we see that $\overline{\mathcal{M}\left(\mathcal{S}_{f}\right)}$ is homeomorphic to $\overline{\mathbb{D}}$, and as observed in [8], the order structure of $\Sigma_{\mathrm{FP}}$ is that of a union of continuous nests which meet only at 0 and $I$ and $\Sigma_{\mathrm{FP}}$ is homeomorphic to a 2-sphere on which the Fourier-Plancherel transform $F$ acts as a quarter-rotation. In particular, $\Sigma_{\mathrm{FP}} \backslash\{0, I\}$ is a locally unitary subspace manifold: we clearly have a locally unitary 
structure on $\mathcal{M}\left(\mathcal{S}_{f}\right) \cup \mathcal{M}\left(\mathcal{S}_{f}\right)^{\perp}$, and we can use $F$ to transfer this structure to the remaining subspaces.

Example 4.10. Let $f$ be the admissible pair $f=\left(x^{-1}, x\right)$. An easy extension of [8], Lemma 5.1 shows that every projection $P \in \mathcal{M}\left(\mathcal{S}_{f}\right)$ lies in a continuum of non-commuting continuous nests which intersect only in $\{0, P, I\}$. A simple calculation reveals that the equivalence relation $\sim$ has only two non-trivial equivalence classes and these are antipodal closed quarter-circles, so $\overline{\mathbb{B}^{2}} / \sim$ is homeomorphic to $\overline{\mathbb{B}^{2}}$ and the boundary projections are

$$
\left\{P, P^{\perp}: P=\left[L^{2}((-a, a))\right], a \in[0, \infty]\right\} .
$$

Now although $\Sigma\left(\mathcal{S}_{f}\right)$ is homeomorphic to a 2 -sphere, $\Sigma\left(\mathcal{S}_{f}\right) \backslash\{0, I\}$ is not locally unitary. For if there were a strong operator topology neighbourhood of $P_{0}=\left[L^{2}((-a, a))\right]$ of the form

$$
\mathcal{N}=\left\{\left[\rho(\lambda) P_{0} L^{2}(\mathbb{R})\right]: \lambda \in \mathbb{B}^{2}\right\}
$$

for some unitary-valued representation $\rho$ of $\mathbb{R}^{2}$, then $\mathcal{N}$ would intersect $\mathcal{M}\left(\mathcal{S}_{f}\right)$ and so contain a projection $P=\left[\rho(\lambda) P_{0} L^{2}(\mathbb{R})\right]$ for some point $\lambda \in \mathbb{B}^{2}$ such that every neighbourhood of $P$ contains two noncommuting projections which are comparable with $P$. Applying $\rho(-\lambda)$, we see that $\mathcal{N}$ must contain two non-commuting projections which are comparable with $P_{0}$. However, all the projections in $\Sigma\left(\mathcal{S}_{f}\right)$ which are comparable with $P_{0}$ commute since by the F. \& M. Riesz theorem, they are of the form $\left[L^{2}(E)\right]$ for some $E \subseteq \mathbb{R}$.

Example 4.11. If we take $f=\left(\frac{1}{3} x^{3}, \frac{1}{2} x^{2}, x\right)$ then $f^{\prime}=\left(x^{2}, x, 1\right)$ and it is not hard to check that the corresponding equivalence relation $\sim$ on $\overline{\mathbb{B}^{3}}$ has two non-trivial equivalence classes:

$$
\begin{array}{r}
\{(0,0,1)\} \cup\left\{(a, b, c) \in S^{2}: b^{2} \leq 4 a c, a>0\right\} \quad \text { and } \\
\{(0,0,-1)\} \cup\left\{(a, b, c) \in S^{2}: b^{2} \leq 4 a c, a<0\right\}
\end{array}
$$

which correspond to 0 and $I$ respectively when we identify the quotient space $\overline{\mathbb{B}^{3}} / \sim$ with $\overline{\mathcal{M}\left(\mathcal{S}_{f}\right)}$. These equivalence classes are closed and so $\overline{\mathcal{M}\left(\mathcal{S}_{f}\right)}$ is homeomorphic to $\overline{\mathbb{B}^{3}}$. If $P$ is a projection in $\mathcal{M}\left(\mathcal{S}_{f}\right)$ then we claim that the projections $\left[e^{i k x} P L^{2}(\mathbb{R})\right]$ for $k \in \mathbb{R}$ are the only non-trivial elements of $\overline{\mathcal{M}\left(\mathcal{S}_{f}\right)}$ which are comparable with $P$. To see this, recall that no proper subspace of the form $L^{2}(E)$ is comparable with $P L^{2}(\mathbb{R})$ by the F. \& M. Riesz theorem, and if $e^{i\langle f, \lambda\rangle} H^{2}(\mathbb{R}) \subseteq$ $e^{i\langle f, \mu\rangle} H^{2}(\mathbb{R})$ and $\lambda \neq \mu$ then $e^{i\langle f, \lambda-\mu\rangle} H^{2}(\mathbb{R}) \subseteq H^{2}(\mathbb{R})$ and so $e^{i\langle f, \lambda-\mu\rangle}$ is a nonzero continuous inner function. This must be of the form $\alpha e^{i \beta x}$ for a unimodular constant $\alpha$ and $\beta \in \mathbb{R}$ (see [6]), which verifies the claim. On the other hand, the boundary of $\overline{\mathcal{M}\left(\mathcal{S}_{f}\right)}$ consists of the projections $\left[L^{2}(E)\right]$ where $E$ is either an interval or the complement of an interval. As in the previous example, it follows that the topological 3-sphere 
$\Sigma\left(\mathcal{S}_{f}\right)$ cannot be locally unitary away from $\{0, I\}$ since the local order structure changes on the boundary of $\overline{\mathcal{M}\left(\mathcal{S}_{f}\right)}$.

Example 4.12. Let $f=\left(x, \log |x|,-x^{-1}\right)$. Then $f^{\prime}=\left(1, x^{-1}, x^{-2}\right)$ so if $\lambda=(a, b, c)$ then $\operatorname{sgn}\left\langle f^{\prime}, \lambda\right\rangle=\operatorname{sgn}\left(a x^{2}+b x+c\right)$. The equivalence relation for $f$ on $\overline{\mathbb{B}^{3}}$ is therefore identical to the relation considered in the previous example, and $\overline{\mathcal{M}\left(\mathcal{S}_{f}\right)}$ is again homeomorphic to $\overline{\mathbb{B}^{3}}$. The order structure differs however, since $\overline{\mathcal{M}\left(\mathcal{S}_{f}\right)}$ contains the set $\overline{\mathcal{M}\left(\mathcal{S}_{\left(x, x^{-1}\right)}\right)}$ from Example 4.10. We call the set $\Sigma_{\text {hyp }}=\Sigma\left(\mathcal{S}_{f}\right)$ the hyperbolic sphere. This was first considered in [8], Section 7. We remark that as in Example $4.10, \Sigma_{\text {hyp }} \backslash\{0, I\}$ cannot be locally unitary.

We can now easily establish the compactness of the "extended hyperbolic lattice" $\hat{\mathcal{L}}$ considered in [8]; this fact was alluded to but not proven there. For $\theta \in \mathbb{T}$, let $u_{\theta}: \mathbb{R} \rightarrow \mathbb{C}$ be the two-valued function taking the value 1 on $[0, \infty)$ and $\theta$ on $(-\infty, 0)$. Then $\hat{\mathcal{L}}$ may be succinctly described as the set of projections

$$
\hat{\mathcal{L}}=\bigcup_{\theta \in \mathbb{T}} \operatorname{Ad}\left(M_{u_{\theta}}\right) \Sigma_{\text {hyp }} .
$$

Now $\Sigma_{\text {hyp }}$ is homeomorphic to the compact space $S^{3}$ and $\hat{\mathcal{L}}$ is a continuous image of $\mathbb{T} \times \Sigma_{\text {hyp }}$ which is compact, so $\hat{\mathcal{L}}$ is also compact.

Example 4.13. The equivalence relation $\sim$ need not have a finite number of nontrivial equivalence classes. For example, consider the admissible triple $f=\left(\frac{1}{2} x^{2}, \log |x|,-x^{-1}\right)$ so that if $\lambda=(a, b, c)$ then $\operatorname{sgn}\left\langle f^{\prime}, \lambda\right\rangle=\operatorname{sgn}\left(a x^{3}+b x+c\right)$. A simple analysis of cases reveals that the nontrivial equivalence classes for $\sim$ in the upper hemisphere $\left\{(a, b, c) \in S^{2}: a \geq 0\right\}$ are

$$
I_{t}=\left\{\lambda /\|\lambda\|: \lambda=\left(1, s,-t\left(s+t^{2}\right)\right), s \geq-3 t^{2} / 4\right\} \cup\left\{\mu_{t}\right\}, \quad t \in \mathbb{R}
$$

where $\mu_{t}=\left(1+t^{2}\right)^{-1 / 2}(0,1,-t)$. The class $I_{t}$ corresponds to the projection $\left[L^{2}(-\infty, t)\right] \in \overline{\mathcal{M}\left(\mathcal{S}_{f}\right)}$. Since points of the form $\left(1, s,-t\left(s+t^{2}\right)\right)$ for $s \in \mathbb{R}$ form a straight line in the plane $\{(1, b, c): b, c \in \mathbb{R}\}$ it follows that $I_{t}$ is the geodesic on $S^{2}$ joining $\lambda_{t} /\left\|\lambda_{t}\right\|$ to $\mu_{t}$ where $\lambda_{t}=\left(1,-3 t^{2} / 4,-t^{3} / 4\right)$. In the $a \leq 0$ hemisphere the nontrivial equivalence classes are the sets $-I_{t}$ which correspond to $\left[L^{2}(t, \infty)\right]$. It is easy to see that the quotient space $\overline{\mathbb{B}^{3}} / \sim$ is homeomorphic to $\overline{\mathbb{B}^{3}}$; indeed, we may choose a homeomorphism which contracts each geodesic $I_{t}$ to the point $\mu_{t}$ and extend this to all of $\overline{\mathbb{B}^{3}}$ is a straightforward manner. We again conclude that $\overline{\mathcal{M}\left(\mathcal{S}_{f}\right)}$ is homeomorphic to $\overline{\mathbb{B}^{3}}$.

Remark 4.14. We do not know if $\overline{\mathcal{M}\left(\mathcal{S}_{f}\right)}$, or equivalently $\overline{\mathbb{B}^{n}} / \sim$, is homeomorphic to $\overline{\mathbb{B}^{n}}$ for every admissible $n$-tuple $f$. It is natural to try to emulate the argument of Proposition 4.8, and it is not hard to show that the equivalence classes $[\lambda]$ of $\sim$ satisfy the following conditions:

(i) $[\lambda]=\{\lambda\}$ if $\lambda \in \mathbb{B}^{n}$; 
(ii) $[-\lambda]=-[\lambda]$ and $[-\lambda] \cap[\lambda]=\emptyset$ for $\lambda \in S^{n-1}$;

(iii) if $\lambda \sim \mu$ then $\lambda \sim \nu$ for every $\nu$ on the geodesic in $S^{n-1}$ joining $\lambda$ to $\mu$; and

(iv) if $\lambda_{n} \sim \mu_{n}$ where $\lambda_{n} \rightarrow \lambda$ and $\mu_{n} \rightarrow \mu$ are convergent sequences in $S^{n-1}$, then $\lambda \sim \mu$.

However, for $n>2$ we have been unable to identify the quotient space $\overline{\mathbb{B}^{n}} / \sim$ for such an equivalence relation.

Remark 4.15. The order structure of the 2-sphere $\Sigma_{\mathrm{FP}}$ and the 3sphere $\Sigma_{\text {hyp }}$ can be viewed as providing an inherent foliation. We exploit this structure in the next section in the determination of their unitary automorphism groups. On the other hand we see in Lemma 5.8 that the 2-spheres determined by monomial pairs $x^{p}, x^{q}$, for $|p|,|q|>1$ have a trivial order structure supported in the common boundary of $\mathcal{M}$ and $\mathcal{M}^{\perp}$.

The Fourier-Plancherel sphere seems to be a particularly distinguished example amongst these 2 -spheres. Furthermore its equator, $\Sigma_{\mathrm{e}}$ yields an interesting compact 1-dimensional subspace manifold which is locally unitary and is probably not (periodically) unitary. It would be interesting to determine other (unitarily inequivalent) subspace manifolds of this form.

\section{UNITARY AUTOMORPHISMS AND ISOMORPHISMS}

Given a set of projections $\mathcal{P} \subseteq \operatorname{Proj}(\mathcal{H})$, the unitary automorphism group of $\mathcal{P}$ is

$$
\mathcal{U}(\mathcal{P})=\{U \in \operatorname{Unit}(\mathcal{H}):(\operatorname{Ad} U) \mathcal{P}=\mathcal{P}\} .
$$

In this section we compute the unitary automorphism groups of the Fourier-Plancherel sphere, the hyperbolic sphere and the extended hyperbolic lattice. These projection manifolds inherit a relatively rich order structure from $\operatorname{Proj}\left(L^{2}(\mathbb{R})\right)$ which we are able to exploit. In contrast we show in Section 5.3 that many other polynomial 2-spheres are essentially rigid. Further operator algebra related to the two main examples can be found in [15], [16], [12].

5.1. The Fourier-Plancherel sphere. Recall the definition of the Fourier-Plancherel sphere $\Sigma_{\mathrm{FP}}$ from Example 4.9. The following notation from [7] is convenient:

$$
\begin{gathered}
\varphi_{s} \in L^{\infty}(\mathbb{R}), \quad \varphi_{s}(x)=e^{-i s x^{2} / 2}, \quad s \in \mathbb{R}, \\
V_{t} \in \operatorname{Unit}\left(L^{2}(\mathbb{R})\right), \quad V_{t} f(x)=e^{t / 2} f\left(e^{t} x\right), \quad t \in \mathbb{R} .
\end{gathered}
$$

The set $\Sigma_{\mathrm{FP}}$ contains two nests of particular interest, which we call the analytic nest

$$
\mathcal{N}_{\mathrm{a}}=\left\{\left[M_{e^{i \lambda x}} H^{2}(\mathbb{R})\right]: \lambda \in \mathbb{R}\right\} \cup\{0, I\}
$$




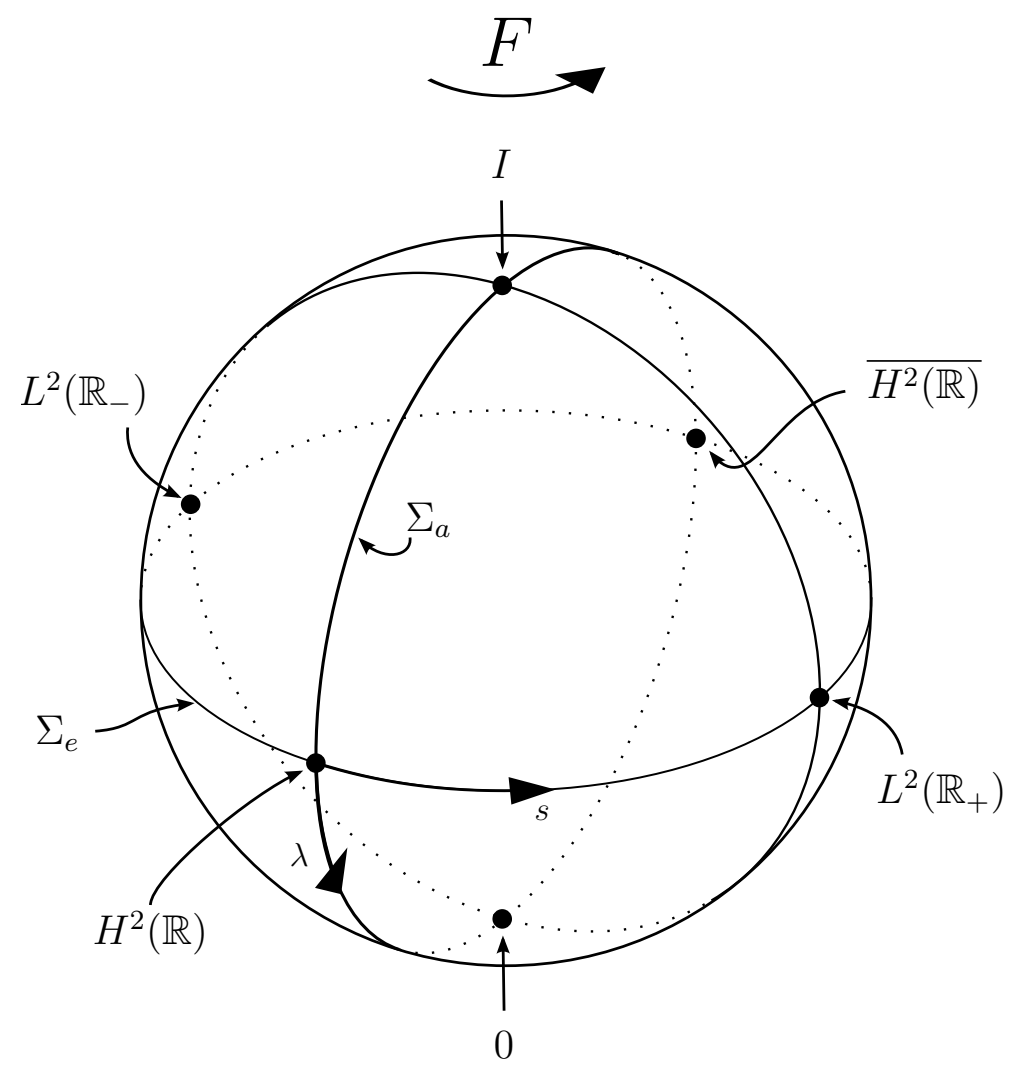

FiguRE 1. A natural realisation of $\Sigma_{\mathrm{FP}}$, the FourierPlancherel sphere, on which the Fourier-Plancherel transform $F$ acts as a quarter-rotation.

and the Volterra nest

$$
\mathcal{N}_{\mathrm{v}}=\left\{\left[L^{2}(t, \infty)\right]: t \in \mathbb{R}\right\} \cup\{0, I\} .
$$

If we denote the nest $\operatorname{Ad}\left(M_{\varphi_{s}}\right) \mathcal{N}_{\mathrm{a}}$ by $\mathcal{N}_{s}$ for $s \in \mathbb{R}$ then

$$
\Sigma_{\mathrm{FP}}=\mathcal{N}_{\mathrm{v}} \cup \mathcal{N}_{\mathrm{v}}^{\perp} \cup \bigcup_{s \in \mathbb{R}}\left(\mathcal{N}_{s} \cup \mathcal{N}_{s}^{\perp}\right),
$$

and the order structure that $\Sigma_{\mathrm{FP}}$ inherits from $\operatorname{Proj}\left(L^{2}(\mathbb{R})\right)$ is such that if $P, Q \in \Sigma_{\mathrm{FP}} \backslash\{0, I\}$ with $P \neq Q$ then $P \vee Q=I$ and $P \wedge Q=0$ unless $\{P, Q\} \subseteq \mathcal{N}$ for some nest $\mathcal{N}$ in this union.

It is easy to see that $M_{\varphi_{s}}, M_{e^{i \lambda x}}$ and $V_{t}$ all lie in $\mathcal{U}\left(\Sigma_{\mathrm{FP}}\right)$ for $s, t, \lambda \in \mathbb{R}$, as does the Fourier-Plancherel transform $F$ since

$$
(\operatorname{Ad} F) \mathcal{N}_{\mathrm{a}}=\mathcal{N}_{\mathrm{v}}, \quad(\operatorname{Ad} F) \mathcal{N}_{s}= \begin{cases}\mathcal{N}_{-1 / s}^{\perp} & s>0 \\ \mathcal{N}_{-1 / s} & s<0\end{cases}
$$

by [8], Theorem 7.1. We first show that $V_{t}$ may be expressed solely in terms of $\left\{M_{\varphi_{s}}: s \in \mathbb{R}\right\}$ and $F$. 
Lemma 5.1. For $t \in \mathbb{R}$, the dilation operator $V_{t}$ lies in the group generated by $\left\{M_{\varphi_{s}}, F, e^{i \psi} I: s, \psi \in \mathbb{R}\right\}$. In fact,

$$
V_{t}=e^{i \pi / 4} M_{\varphi_{\exp (t)}} F M_{\varphi_{\exp (-t)}} F M_{\varphi_{\exp (t)}} F .
$$

Proof. Let us write $S_{g}$ for the operation of convolution with a function $g \in L^{\infty}(\mathbb{R})$, defined on the Schwartz space $\mathcal{S}(\mathbb{R})$; that is,

$$
S_{g} f(x)=\int_{\mathbb{R}} g(x-t) f(t) d t, \quad f \in \mathcal{S}(\mathbb{R}), x \in \mathbb{R} .
$$

For $\zeta \in \mathbb{C} \backslash \mathbb{R}_{-}$, let $\zeta^{ \pm 1 / 2}$ denote the square root of $\zeta^{ \pm 1}$ with nonnegative real part. Let $\tilde{F}$ be the alternate Fourier transform defined on $\mathcal{S}(\mathbb{R})$ by

$$
\tilde{F} f(x)=\int_{\mathbb{R}} f(y) e^{-2 \pi i x y} d y, \quad f \in \mathcal{S}(\mathbb{R}), x \in \mathbb{R} .
$$

Observe that $\tilde{F}=\left.V_{\log 2 \pi} F\right|_{\mathcal{S}(\mathbb{R})}$ and that $V_{t} M_{\varphi_{s}}=M_{\varphi_{e^{2 t} s}} V_{t}$.

In Section XI.1 of [9] it is shown that

$$
\tilde{F} S_{\varphi_{2 \pi b}}=(i b)^{-1 / 2} M_{\varphi_{-2 \pi / b}} \tilde{F}, \quad b \in \mathbb{R} \backslash\{0\},
$$

or, writing $s=2 \pi b$ and rearranging,

$$
S_{\varphi_{s}} f=(2 \pi / i s)^{1 / 2} F^{*} M_{\varphi_{-1 / s}} F f, \quad f \in \mathcal{S}(\mathbb{R}), s \in \mathbb{R} \backslash\{0\} .
$$

Observe that $\varphi_{s}(x-t)=e^{i s x t} \varphi_{s}(x) \varphi_{s}(t)$ for $x, s, t \in \mathbb{R}$. Hence for $x \in \mathbb{R}, s<0$ and $f \in \mathcal{S}(\mathbb{R})$,

$$
\begin{aligned}
S_{\varphi_{s}} f(x) & =\int_{\mathbb{R}} \varphi_{s}(x-t) f(t) d t \\
& =\varphi_{s}(x) \int_{\mathbb{R}} e^{i s x t} \varphi_{s}(t) f(t) d t \\
& =(2 \pi)^{1 / 2} \varphi_{s}(x)\left(F^{*} M_{\varphi_{s}} f\right)(s x) \\
& =(2 \pi /(-s))^{1 / 2} M_{\varphi_{s}} V_{\log (-s)} F M_{\varphi_{s}} f(x) .
\end{aligned}
$$

Equating these expressions for $S_{\varphi_{s}} f$ and using the density of $\mathcal{S}(\mathbb{R})$ in $L^{2}(\mathbb{R})$ gives

$$
V_{\log (-s)}=e^{i \pi / 4} M_{\varphi_{-s}} F^{*} M_{\varphi_{-1 / s}} F M_{\varphi_{-s}} F^{*} .
$$

Now $F^{*}=F^{3}$ and $F^{2}$ commutes with $M_{\varphi_{\sigma}}$ for any $\sigma \in \mathbb{R}$, since $\varphi_{\sigma}$ is even and $F^{2} f(x)=f(-x)$. So

$$
F M_{\varphi_{\sigma}} F^{*}=F^{*} M_{\varphi_{\sigma}} F \quad \text { and } \quad F^{*} M_{\varphi_{\sigma}} F^{*}=F M_{\varphi_{\sigma}} F .
$$

Using this and setting $t=\log (-s)$ completes the proof.

Theorem 5.2. The unitary automorphism group of $\Sigma_{\mathrm{FP}}$ is generated by

$$
\left\{M_{\varphi_{s}}, M_{e^{i \lambda x}}, F, e^{i \psi} I: s, \lambda, \psi \in \mathbb{R}\right\}
$$


Proof. Let $U \in \mathcal{U}\left(\Sigma_{\mathrm{FP}}\right)$ and let $\Sigma_{\mathrm{a}}, \Sigma_{\mathrm{v}}$ and $\Sigma_{s}$ denote the "great circles"

$$
\Sigma_{\mathrm{a}}=\mathcal{N}_{\mathrm{a}} \cup \mathcal{N}_{\mathrm{a}}^{\perp}, \quad \Sigma_{\mathrm{v}}=\mathcal{N}_{\mathrm{v}} \cup \mathcal{N}_{\mathrm{v}}^{\perp}, \quad \Sigma_{s}=\mathcal{N}_{s} \cup \mathcal{N}_{s}^{\perp} \text { for } s \in \mathbb{R} .
$$

The map Ad $U$ preserves orthogonality and the order structure on $\Sigma_{\mathrm{FP}}$, so it must permute these great circles. If $(\operatorname{Ad} U) \Sigma_{\mathrm{a}}=\Sigma_{\mathrm{v}}$, then $\operatorname{Ad} F U$ fixes $\Sigma_{\mathrm{a}}$; if $(\operatorname{Ad} U) \Sigma_{\mathrm{a}}=\Sigma_{s}$ for some $s \in \mathbb{R}$, then $M_{\varphi_{-s}} U$ fixes $\Sigma_{\mathrm{a}}$. So we may assume that $(\operatorname{Ad} U) \Sigma_{\mathrm{a}}=\Sigma_{\mathrm{a}}$.

If $(\operatorname{Ad} U) \Sigma_{\mathrm{v}} \neq \Sigma_{\mathrm{v}}$, then $(\operatorname{Ad} U) \Sigma_{\mathrm{v}}=\Sigma_{s}$ for some $s \neq 0$. If $s>0$ then by (1), $(\operatorname{Ad} F) \Sigma_{s}=\Sigma_{-1 / s}$ and $(\operatorname{Ad} F) \Sigma_{\mathrm{a}}=\Sigma_{\mathrm{v}}$, so $U^{\prime}=F^{*} M_{\varphi_{1 / s}} F U$ satisfies $\left(\operatorname{Ad} U^{\prime}\right) \Sigma_{\mathrm{a}}=\Sigma_{\mathrm{a}}$ and $\left(\operatorname{Ad} U^{\prime}\right) \Sigma_{\mathrm{v}}=\Sigma_{\mathrm{v}}$. So we may assume that $(\operatorname{Ad} U) \Sigma_{\mathrm{v}}=\Sigma_{\mathrm{v}}$ and $(\operatorname{Ad} U) \Sigma_{\mathrm{a}}=\Sigma_{\mathrm{a}}$.

There are now four cases to consider:

(i) $(\operatorname{Ad} U) \mathcal{N}_{\mathrm{a}}=\mathcal{N}_{\mathrm{a}},(\operatorname{Ad} U) \mathcal{N}_{\mathrm{v}}=\mathcal{N}_{\mathrm{v}}$;

(ii) $(\operatorname{Ad} U) \mathcal{N}_{\mathrm{a}}=\mathcal{N}_{\mathrm{a}}^{\perp},(\operatorname{Ad} U) \mathcal{N}_{\mathrm{v}}=\mathcal{N}_{\mathrm{v}}^{\perp}$;

(iii) $(\operatorname{Ad} U) \mathcal{N}_{\mathrm{a}}=\mathcal{N}_{\mathrm{a}}^{\perp},(\operatorname{Ad} U) \mathcal{N}_{\mathrm{v}}=\mathcal{N}_{\mathrm{v}}$;

(iv) $(\operatorname{Ad} U) \mathcal{N}_{\mathrm{a}}=\mathcal{N}_{\mathrm{a}},(\operatorname{Ad} U) \mathcal{N}_{\mathrm{v}}=\mathcal{N}_{\mathrm{v}}^{\perp}$.

Replacing $U$ with $F^{2} U$ interchanges cases (i) and (ii) and also interchanges cases (iii) and (iv), so it suffices to consider cases (i) and (iii) only.

Suppose that case (iii) holds. We claim that $(\operatorname{Ad} U) \mathcal{N}_{1}=\mathcal{N}_{-s}^{\perp}$ for some $s>0$. To see this, let $\mathfrak{N}$ be the set of nests

$$
\mathfrak{N}=\left\{\mathcal{N}_{\mathrm{v}}, \mathcal{N}_{\mathrm{v}}^{\perp}\right\} \cup\left\{\mathcal{N}_{s}, \mathcal{N}_{s}^{\perp}: s \in \mathbb{R}\right\}
$$

so that $\Sigma_{\mathrm{FP}}$ is the union of all nests in $\mathfrak{N}$. Since $U$ is unitary, it maps nests onto nests and so induces a bijection of $\mathfrak{N}$.

Let $\Sigma_{\mathrm{e}}$ be the "equator" of $\Sigma_{\mathrm{FP}}$,

$$
\Sigma_{\mathrm{e}}=\left\{L^{2}\left(\mathbb{R}_{+}\right), L^{2}\left(\mathbb{R}_{-}\right)\right\} \cup\left\{M_{\varphi_{s}} H^{2}(\mathbb{R}), M_{\varphi_{s}} \overline{H^{2}(\mathbb{R})}: s \in \mathbb{R}\right\} .
$$

Here $\overline{H^{2}(\mathbb{R})}$ is the set of complex conjugates of functions in $H^{2}(\mathbb{R})$, which is equal to $H^{2}(\mathbb{R})^{\perp}[13]$. The set $\Sigma_{\mathrm{e}}$ contains exactly one subspace from each nest in $\mathfrak{N}$, so the action $(\operatorname{Ad} U): \Sigma_{\mathrm{e}} \rightarrow(\operatorname{Ad} U) \Sigma_{\mathrm{e}}, K \mapsto$ $(\operatorname{Ad} U) K$ of $\operatorname{Ad} U$ on $\Sigma_{\mathrm{e}}$ determines the action of $\operatorname{Ad} U$ on $\mathfrak{N}$. Moreover, $\operatorname{Ad} U$ is a homeomorphism between $\Sigma_{\mathrm{e}}$ and $(\operatorname{Ad} U) \Sigma_{\mathrm{e}}$, and $\Sigma_{\mathrm{e}}$ is itself homeomorphic to the circle $\mathbb{T}$. Let us give $\mathfrak{N}$ the topology induced by the topology on $\Sigma_{\mathrm{e}}$. The bijective action of $\operatorname{Ad} U$ on $\mathfrak{N}$ is then a homeomorphism.

It follows that the closed connected set

$$
\left[\mathcal{N}_{\mathrm{a}}, \mathcal{N}_{\mathrm{v}}\right]=\bigcup_{s \geq 0} \mathcal{N}_{s} \cup \mathcal{N}_{\mathrm{v}}
$$


must be mapped by $\operatorname{Ad} U$ onto

$$
\begin{gathered}
\text { either } \quad\left[\mathcal{N}_{\mathrm{a}}^{\perp}, \mathcal{N}_{\mathrm{v}}\right]=\bigcup_{s \geq 0} \mathcal{N}_{s}^{\perp} \cup \mathcal{N}_{\mathrm{v}}^{\perp} \cup \bigcup_{s \in \mathbb{R}} \mathcal{N}_{s} \cup \mathcal{N}_{\mathrm{v}} \\
\quad \text { or } \quad\left[\mathcal{N}_{\mathrm{v}}, \mathcal{N}_{\mathrm{a}}^{\perp}\right]=\mathcal{N}_{\mathrm{v}} \cup \bigcup_{s \leq 0} \mathcal{N}_{s}^{\perp}
\end{gathered}
$$

If $(\operatorname{Ad} U)\left[\mathcal{N}_{\mathrm{a}}, \mathcal{N}_{\mathrm{v}}\right]=\left[\mathcal{N}_{\mathrm{a}}^{\perp}, \mathcal{N}_{\mathrm{v}}\right]$ then there is some $s>0$ such that $(\operatorname{Ad} U) \mathcal{N}_{s}=\mathcal{N}_{\mathrm{v}}^{\perp}$. Since $(\operatorname{Ad} U) \mathcal{N}_{\mathrm{v}}=\mathcal{N}_{\mathrm{v}}$ and $U$ is unitary,

$$
\mathcal{N}_{\mathrm{v}}^{\perp}=\left(\left(\operatorname{Ad} U^{*}\right) \mathcal{N}_{\mathrm{v}}\right)^{\perp}=\left(\operatorname{Ad} U^{*}\right) \mathcal{N}_{\mathrm{v}}^{\perp}=\mathcal{N}_{s}
$$

which is impossible by the F. \& M. Riesz theorem.

So $(\operatorname{Ad} U)\left[\mathcal{N}_{\mathrm{a}}, \mathcal{N}_{\mathrm{v}}\right]=\left[\mathcal{N}_{\mathrm{v}}, \mathcal{N}_{\mathrm{a}}^{\perp}\right]$ and so $(\operatorname{Ad} U) \mathcal{N}_{1}=\mathcal{N}_{-s}^{\perp}$ for some $s>0$. Since $(\operatorname{Ad} U) \mathcal{N}_{\mathrm{v}}=\mathcal{N}_{\mathrm{v}}$, it follows from [3], Chapter 17 that there exist a unimodular function $\alpha \in L^{\infty}(\mathbb{R})$ and an order-preserving almost everywhere differentiable bijection $g: \mathbb{R} \rightarrow \mathbb{R}$ such that $U=M_{\alpha} C_{g}$ where $C_{g}$ is the unitary composition operator corresponding to $g$. Thus

$$
U M_{\varphi_{1}} H^{2}(\mathbb{R})=M_{\alpha} C_{g} M_{\varphi_{1}} H^{2}(\mathbb{R})=M_{\varphi_{-s}} M_{e^{i \lambda x}} \overline{H^{2}(\mathbb{R})}
$$

for some $\lambda \in \mathbb{R}$. Moreover, $(\operatorname{Ad} U) \mathcal{N}_{\mathrm{a}}=\mathcal{N}_{\mathrm{a}}^{\perp}$, so $U H^{2}(\mathbb{R})=M_{e^{i \mu x}} \overline{H^{2}(\mathbb{R})}$ for some $\mu \in \mathbb{R}$. Since $C_{g} M_{f}=M_{f \circ g} C_{g}$ for $f \in L^{\infty}(\mathbb{R})$,

$$
M_{\varphi_{1} \circ g} M_{\alpha} C_{g} H^{2}(\mathbb{R})=M_{\varphi_{1} \circ g} M_{e^{i \mu x}} \overline{H^{2}(\mathbb{R})}=M_{\varphi_{-s}} M_{e^{i \lambda x}} \overline{H^{2}(\mathbb{R})} .
$$

Taking orthogonal complements, we see that

$$
M_{\varphi_{s}} M_{e^{-i \lambda x}} M_{\varphi_{1} \circ g} M_{e^{i \mu x}} H^{2}(\mathbb{R})=u H^{2}(\mathbb{R})=H^{2}(\mathbb{R}),
$$

where $u: \mathbb{R} \rightarrow \mathbb{C}$ is the unimodular function

$$
x \mapsto \exp i\left(-\frac{1}{2}\left(g(x)^{2}+s x^{2}\right)+(\mu-\lambda) x\right) .
$$

So $u$ must be constant almost everywhere. But $s>0$ and $g(x) \rightarrow \infty$ as $x \rightarrow \infty$, so this is impossible.

So we are reduced to case (i): Ad $U$ fixes both the analytic nest and the Volterra nest, and so is a unitary automorphism of $\operatorname{Alg}\left(\mathcal{N}_{\mathrm{v}} \cup \mathcal{N}_{\mathrm{a}}\right)$, the Fourier binest algebra. By [7], Lemma 4.1, $U=e^{i \psi} M_{e^{i \lambda x}} D_{\mu} V_{t}$ for some $\psi, \lambda, \mu, t \in \mathbb{R}$. Now apply Lemma 5.1.

Remark 5.3. It can be shown that, modulo scalars, this automorphism group is isomorphic to the semidirect product $\mathbb{R}^{2} \rtimes S L_{2}(\mathbb{R})$. The isomorphism is implemented by the map sending

$$
\left[\begin{array}{ccc}
1 & & \\
\lambda & 1 & 0 \\
\mu & 0 & 1
\end{array}\right], \quad\left[\begin{array}{ccc}
1 & & \\
& 1 & -s \\
& 0 & 1
\end{array}\right] \text { and }\left[\begin{array}{ccc}
1 & & \\
& 0 & -1 \\
& 1 & 0
\end{array}\right]
$$

to $\operatorname{Ad}\left(M_{e^{i \lambda x}} D_{\mu}\right), \operatorname{Ad}\left(M_{\varphi_{s}}\right)$ and $\operatorname{Ad}(F)$ respectively. We refer the reader to [11] for the details. 
It is perhaps surprising that $\left\{\operatorname{Ad}(U): U \in \mathcal{U}\left(\Sigma_{\mathrm{FP}}\right)\right\}$ has such a simple description. The authors do not know if the same can be said for $\mathcal{U}\left(\Sigma_{\mathrm{FP}}\right)$ itself.

\subsection{The hyperbolic sphere and the extended hyperbolic lat-}

tice. Recall the definitions of the hyperbolic sphere $\Sigma_{\text {hyp }}$ and the extended hyperbolic lattice $\hat{\mathcal{L}} \supseteq \Sigma_{\text {hyp }}$ from Example 4.12. For $\lambda, \mu \in \mathbb{R}$, let $M_{\lambda, \mu}=M_{e^{i\left(\lambda x+\mu x^{-1}\right)}}$ and for $(\theta, s) \in \mathbb{T} \times \mathbb{R}$ let

$$
U_{\theta, s}=M_{|x|^{i s} u_{\theta}(x)} \text { where as before, } u_{\theta}=\chi_{[0, \infty)}+\theta \chi_{(-\infty, 0)} .
$$

A typical projection in $\mathcal{M}\left(\mathcal{S}_{\left(x, \log |x|,-x^{-1}\right)}\right)$ is

$$
\left[U_{1, s} M_{\lambda, \mu} H^{2}(\mathbb{R})\right] \quad \text { where }(s, \lambda, \mu) \in \mathbb{R}^{3} .
$$

If $u_{\theta, s}=\chi_{[0, \infty)}+\theta e^{s \pi} \chi_{(-\infty, 0)}$ then it is shown in [8] that $U_{\theta, s} H^{2}(\mathbb{R})=$ $M_{u_{\theta, s}} H^{2}(\mathbb{R})$ for $(\theta, s) \in \mathbb{T} \times \mathbb{R}$. We further define operators

$$
J_{1} f(x)=x^{-1} f\left(-x^{-1}\right) \quad \text { and } \quad J_{2} f(x)=f(-x), \quad f \in L^{2}(\mathbb{R}) ;
$$

these are the unitary composition operators corresponding to the symmetries $x \mapsto-x^{-1}$ and $x \mapsto-x$, respectively. The linear span of the set of functions $z \mapsto(z-\xi)^{-1}$ for $\Im \xi<0$ (or $\Im \xi>0$ ) is dense in $H^{2}(\mathbb{R})$ (or in $\overline{H^{2}(\mathbb{R})}$, respectively). Applying $J_{1}$ and $J_{2}$ to these sets reveals that $J_{1} H^{2}(\mathbb{R})=H^{2}(\mathbb{R})$ and $J_{2} H^{2}(\mathbb{R})=\overline{H^{2}(\mathbb{R})}$. It is easy to see that all of these operators are unitary automorphisms of $\hat{\mathcal{L}}$, and if we fix $\theta=1$ then we obtain unitary automorphisms of $\Sigma_{\text {hyp }}$. We will show that in each case, these operators generate the whole unitary automorphism group.

Lemma 5.4. Let $\gamma$ be a conformal automorphism of the upper half plane $\mathbb{H}$. For each nonzero $s \in \mathbb{R}$, the subspace $M_{u_{1, s} \circ \gamma} H^{2}(\mathbb{R})$ has zero intersection with each subspace in $\mathcal{M}\left(\mathcal{S}_{\left(x, \log |x|,-x^{-1}\right)}\right)$ unless $\gamma$ is either of the form $\gamma(x)=$ ax for some $a>0$ or $\gamma(x)=-b x^{-1}$ for some $b>0$.

Proof. Suppose that $M_{u_{1, s} \circ \gamma} H^{2}(\mathbb{R}) \cap U_{1, \sigma} M_{\lambda, \mu} H^{2}(\mathbb{R}) \neq\{0\}$. Let

$$
f=\left(u_{1, s} \circ \gamma\right) g=u_{1, \sigma} e^{i\left(\lambda x+\mu x^{-1}\right)} h
$$

be a nonzero function in this intersection, where $g, h \in H^{2}(\mathbb{R})$. Multiplying this equation by $e^{-i \lambda x}$ if $\lambda<0$ and by $e^{-i \mu x^{-1}}$ if $\mu>0$ and writing $\alpha=\left(u_{1, s} \circ \gamma\right) / u_{1, \sigma}$ gives $\alpha \varphi=\psi$ for nonzero functions $\varphi, \psi \in H^{2}(\mathbb{R})$. Observe that $\alpha$ takes at most four values, since

$$
\alpha(x)= \begin{cases}1 & x \in \gamma^{-1}\left(\mathbb{R}_{+}\right) \cap \mathbb{R}_{+} \\ e^{s \pi} & x \in \gamma^{-1}\left(\mathbb{R}_{-}\right) \cap \mathbb{R}_{+} \\ e^{-\sigma \pi} & x \in \gamma^{-1}\left(\mathbb{R}_{+}\right) \cap \mathbb{R}_{-} \\ e^{(s-\sigma) \pi} & x \in \gamma^{-1}\left(\mathbb{R}_{-}\right) \cap \mathbb{R}_{-}\end{cases}
$$

Applying the F. \& M. Riesz theorem to the function $\alpha \varphi-\psi=0$ reveals that $\alpha$ must be constant almost everywhere; in particular, since $s \neq 0$, no three of these intersections can have nonzero Lebesgue measure. 
Since $\gamma$ induces a conformal automorphism of the upper half plane, either $\gamma(x)=a x+b$ with $a>0$ and $b \in \mathbb{R}$ or $\gamma(x)=a-b(x-c)^{-1}$ with $a, b, c \in \mathbb{R}$ and $b>0$. Applying the condition in the previous paragraph forces $\gamma(x)=a x$ with $a>0$ or $\gamma(x)=-b x^{-1}$ with $b>0$.

Theorem 5.5. (i) The unitary automorphism group of $\hat{\mathcal{L}}$ is equal to the union $G \cup G J_{1} \cup G J_{2} \cup G J_{1} J_{2}$ where

$$
G=\left\{\alpha U_{\theta, s} M_{\lambda, \mu} V_{t}:(\alpha, \theta, s, \lambda, \mu, t) \in \mathbb{T}^{2} \times \mathbb{R}^{4}\right\} .
$$

(ii) The unitary automorphism group of $\Sigma_{\mathrm{hyp}}$ is equal to the union $G_{0} \cup G_{0} J_{1} \cup G_{0} J_{2} \cup G_{0} J_{1} J_{2}$ where

$$
G_{0}=\left\{\alpha U_{1, s} M_{\lambda, \mu} V_{t}:(\alpha, s, \lambda, \mu, t) \in \mathbb{T} \times \mathbb{R}^{4}\right\} .
$$

Proof. (i) We exploit the order structure of $\hat{\mathcal{L}}$, given in Proposition 5.2 of [8]. Suppose that $U$ lies in $\mathcal{U}(\hat{\mathcal{L}})$, the unitary automorphism group of $\hat{\mathcal{L}}$. Let us write $\mathcal{M}$ for the set

$$
\mathcal{M}=\mathcal{M}\left(\mathcal{S}_{\left(x, \log |x|,-x^{-1}\right)}\right)=\left\{\operatorname{Ad}\left(U_{1, s} M_{\lambda, \mu}\right)\left[H^{2}(\mathbb{R})\right]: s, \lambda, \mu \in \mathbb{R}\right\}
$$

and let $\partial \mathcal{M}$ be the topological boundary of $\mathcal{M}$, which by Theorem 4.2 is the set of projections in $\hat{\mathcal{L}}$ of the form $\left[L^{2}(E)\right]$. Observe first that $\partial \mathcal{M}$ must be mapped onto itself by $\operatorname{Ad} U$. This will follow if we can show that the set $\hat{\mathcal{L}} \backslash \partial \mathcal{M}$ may be intrinsically described as the union of all non-commutative sublattices of $\hat{\mathcal{L}}$ which are order-isomorphic to the slice $\mathcal{L}_{1,0}=\left\{\operatorname{Ad}\left(M_{\lambda, \mu}\right)\left[H^{2}(\mathbb{R})\right]: \lambda, \mu \in \mathbb{R}\right\}$ and whose closure contains $\{0, I\}$. Writing

$$
\mathcal{L}_{\theta, s}=\left\{\operatorname{Ad}\left(U_{\theta, s} M_{\lambda, \mu}\right)\left[H^{2}(\mathbb{R})\right]: \lambda, \mu \in \mathbb{R}\right\}
$$

for $(\theta, s) \in \mathbb{T} \times \mathbb{R}$, observe that each of the slices $\mathcal{L}_{\theta, s}, \mathcal{L}_{\theta, s}^{\perp}$ has this property. Hence this union contains $\hat{\mathcal{L}} \backslash \partial \mathcal{M}$. On the other hand, suppose that $\mathcal{L}$ is such a non-commutative sublattice and that $P \in$ $\partial \mathcal{M} \cap \mathcal{L}$. Since $\mathcal{L} \cong \mathcal{L}_{1,0}$ there are two continuous nests $\mathcal{N}_{1}, \mathcal{N}_{2}$ contained in $\mathcal{L} \cup\{0, I\}$ which do not commute with one another such that $\mathcal{N}_{1} \cap \mathcal{N}_{2}=\{0, P, I\}$. Now $P$ is of the form $P=\left[L^{2}(E)\right]$ and all the non-trivial projections $Q, R$ in $\hat{\mathcal{L}}$ which satisfy $Q \leq\left[L^{2}(E)\right] \leq R$ are all contained in $\partial \mathcal{M}$ by the F. \& M. Riesz theorem, so $\mathcal{N}_{1}, \mathcal{N}_{2} \subseteq \partial \mathcal{M}$. But all projections in $\partial \mathcal{M}$ commute, so we obtain a contradiction and $\partial \mathcal{M} \cap \mathcal{L}$ must be empty.

Hence $(\operatorname{Ad} U) \partial \mathcal{M}=\partial \mathcal{M}$. Observe that

$$
\hat{\mathcal{L}} \backslash \partial \mathcal{M}=\bigcup_{\theta \in \mathbb{T}}\left(\operatorname{Ad} U_{\theta, 1}\right) \mathcal{M} \cup \bigcup_{\theta \in \mathbb{T}}\left(\operatorname{Ad} U_{\theta, 1}\right) \mathcal{M}^{\perp}
$$

and that the terms in this union are the components of $\hat{\mathcal{L}} \backslash \partial \mathcal{M}$, which $\operatorname{Ad} U$ must therefore permute. If $U H^{2}(\mathbb{R})=U_{\theta, s} M_{\lambda, \mu} \overline{H^{2}(\mathbb{R})}$, then we may replace $U$ with $J_{2} U$ to ensure that $U H^{2}(\mathbb{R}) \in \bigcup_{\theta \in \mathbb{T}}\left(\operatorname{Ad} U_{\theta, 1}\right) \mathcal{M}$, and then replacing $U$ by $U_{\theta, s} M_{\lambda, \mu} U$ for suitable $\theta, s, \lambda, \mu$ we can arrange that $U H^{2}(\mathbb{R})=H^{2}(\mathbb{R})$, and so also that $(\operatorname{Ad} U) \mathcal{M}=\mathcal{M}$. 
TABLE 1. Commutation relations for $\mathcal{U}(\hat{\mathcal{L}})$

\begin{tabular}{rr|ccccc} 
& \multicolumn{5}{|c}{$Y$} & \\
& $X Y$ & $U_{\theta, s}$ & $M_{\lambda, \mu}$ & $V_{t}$ & $J_{1}$ & $J_{2}$ \\
\hline & $U_{\theta^{\prime}, s^{\prime}}$ & $U_{\theta \theta^{\prime}, s+s^{\prime}}$ & & & & \\
$M_{\lambda^{\prime}, \mu^{\prime}}$ & $c o m m u t e$ & $M_{\lambda+\lambda^{\prime}, \mu+\mu^{\prime}}$ & & & \\
$X$ & $V_{t^{\prime}}$ & $e^{i s t^{\prime}} U_{\theta, s} V_{t^{\prime}}$ & $M_{e^{t^{\prime}} \lambda, e^{-t^{\prime}} \mu} V_{t^{\prime}}$ & $V_{t+t^{\prime}}$ & & \\
& $J_{1}$ & $U_{\bar{\theta},-s} J_{1}$ & $M_{-\mu,-\lambda} J_{1}$ & $V_{-t} J_{1}$ & $I$ & \\
& $J_{2}$ & $U_{\bar{\theta}, s} J_{2}$ & $M_{-\lambda,-\mu} J_{2}$ & commute & commute & $I$
\end{tabular}

Since $(\operatorname{Ad} U) \partial \mathcal{M}=\partial \mathcal{M}, \operatorname{Ad} U$ maps projections in $(\partial \mathcal{M})^{\prime \prime}$ to projections in $(\partial \mathcal{M})^{\prime \prime}$ and so induces an automorphism of $L^{\infty}(\mathbb{R})$. Von Neumann's theorem of [20] shows that this is necessarily induced by a Borel isomorphism $\gamma$. (See also Nordgren [14]). Since $L^{\infty}(\mathbb{R})$ is maximal abelian it follows readily that $U=M_{\varphi} C_{\gamma}$ for some unimodular function $\varphi$ where $C_{\gamma}$ is the unitary composition operator for $\gamma$. Moreover, $U$ induces an automorphism of $H^{\infty}(\mathbb{R})$, since if $h \in H^{\infty}(\mathbb{R})$ then $M_{h} H^{2}(\mathbb{R}) \subseteq H^{2}(\mathbb{R})$ and so

$$
\begin{aligned}
H^{2}(\mathbb{R})=U H^{2}(\mathbb{R}) \supseteq U M_{h} H^{2}(\mathbb{R}) & =M_{\varphi} C_{\gamma} M_{h} H^{2}(\mathbb{R}) \\
& =M_{h \circ \gamma} M_{\varphi} C_{\gamma} H^{2}(\mathbb{R}) \\
& =M_{h \circ \gamma} U H^{2}(\mathbb{R}) \\
& =M_{h \circ \gamma} H^{2}(\mathbb{R}) .
\end{aligned}
$$

It follows from the Beurling-Lax theorem [10] that $h \circ \gamma \in H^{\infty}(\mathbb{R})$. The same argument applied to $U^{*}$ shows that the map $h \mapsto h \circ \gamma$ is indeed an automorphism of $H^{\infty}(\mathbb{R})$. Hence $\gamma$ induces a conformal automorphism of the upper half plane.

Fix $s \neq 0$. Since $(\operatorname{Ad} U) \mathcal{M}=\mathcal{M}$, the subspace

$$
U\left(U_{1, s} H^{2}(\mathbb{R})\right)=U M_{u_{1, s}} H^{2}(\mathbb{R})=M_{u_{1, s} \circ \gamma} U H^{2}(\mathbb{R})=M_{u_{1, s} \circ \gamma} H^{2}(\mathbb{R})
$$

must lie in $\mathcal{M}$. By Lemma 5.4, either $\gamma(x)=e^{t} x$ or $\gamma(x)=-\left(e^{t} x\right)^{-1}$ for some $t \in \mathbb{R}$. Hence $C_{\gamma} \in\left\{V_{t}, J_{1} V_{t}\right\}$. Multiplying by $C_{\gamma}^{*}$ reduces to the case $U=M_{\varphi}$. Since $C_{\gamma} H^{2}(\mathbb{R})=H^{2}(\mathbb{R})$, we have $M_{\varphi} H^{2}(\mathbb{R})=H^{2}(\mathbb{R})$ and so $\varphi$ is constant almost everywhere.

(ii) Following the first paragraph of the proof of (i) with $\Sigma_{\text {hyp }}$ in place of $\hat{\mathcal{L}}$, we may assume that $U \in \mathcal{U}\left(\Sigma_{\text {hyp }}\right)$ satisfies $(\operatorname{Ad} U) \partial \mathcal{M}=\partial \mathcal{M}$. Now $\Sigma_{\text {hyp }} \backslash \partial \mathcal{M}=\mathcal{M} \cup \mathcal{M}^{\perp}$ has two components, which $\operatorname{Ad} U$ must permute. We may again assume that $(\operatorname{Ad} U) \mathcal{M}=\mathcal{M}$ by multiplying by $J_{2}$ if necessary. The remainder of the proof proceeds as above.

From Table 1, we see that $\operatorname{Ad}(\mathcal{U}(\hat{\mathcal{L}}))$ is isomorphic to the double 
semidirect product

$$
\begin{aligned}
\left(\left(\mathbb{T} \times \mathbb{R}^{3}\right) \rtimes_{\alpha} \mathbb{R}\right) & \rtimes_{\beta}(\mathbb{Z} / 2 \mathbb{Z})^{2}, \\
\alpha(t)(\theta, s, \lambda, \mu) & =\left(\theta, s, e^{t} \lambda, e^{-t} \mu\right), \\
\beta(1,0)(\theta, s, \lambda, \mu, t) & =(\bar{\theta},-s,-\mu,-\lambda,-t), \\
\beta(0,1)(\theta, s, \lambda, \mu, t) & =(\bar{\theta}, s,-\lambda,-\mu, t) .
\end{aligned}
$$

The map sending

$$
\left[\begin{array}{ccccc}
1 & & & & \\
a & 1 & 0 & & \\
s & 0 & 1 & & \\
\lambda & & & e^{t} & 0 \\
\mu & & & 0 & e^{-t}
\end{array}\right],\left[\begin{array}{ccccc}
-1 & & & & \\
& 1 & 0 & & \\
& 0 & 1 & & \\
& & & 0 & 1 \\
& & & 1 & 0
\end{array}\right] \text { and }\left[\begin{array}{ccccc}
-1 & & & & \\
& 1 & 0 & & \\
& 0 & -1 & & \\
& & & 1 & 0 \\
& & & 0 & 1
\end{array}\right]
$$

to $\operatorname{Ad}\left(U_{e^{i a}, s} M_{\lambda, \mu} V_{t}\right), \operatorname{Ad}\left(J_{1}\right)$ and $\operatorname{Ad}\left(J_{2}\right)$ respectively for $a, s, \lambda, \mu, t \in \mathbb{R}$ is a homomorphism onto $\operatorname{Ad}(\mathcal{U}(\hat{\mathcal{L}}))$ with kernel $2 \pi \mathbb{Z}$.

5.3. Polynomial 2-spheres. Finally, we consider isomorphisms between 2-spheres of the form $\Sigma_{m, n}=\Sigma\left(\mathcal{M}_{m, n}\right)$ where

$$
\mathcal{M}_{m, n}=\left\{\left[e^{i\left(\lambda x^{m}+\mu x^{n}\right)} H^{2}(\mathbb{R})\right]: \lambda, \mu \in \mathbb{R}\right\} \quad \text { for } \quad m, n \in \mathbb{Z} \backslash\{0\} .
$$

We write $\partial \mathcal{M}_{m, n}$ for the topological boundary of $\mathcal{M}_{m, n}$. This can be easily computed using the results of Section 4:

Lemma 5.6. Let $m>n$ be nonzero integers and let $\alpha$ be the residue class of $(m, n)$ in $(\mathbb{Z} / 2 \mathbb{Z})^{2}$, which we identify with $\{0,1\} \times\{0,1\}$. Then $\partial \mathcal{M}_{m, n}=\partial \mathcal{M}_{\alpha}$ depends only on $\alpha$. In fact $\partial \mathcal{M}_{(0,1)}=\Sigma_{\mathrm{v}}$, the Volterra circle,

$$
\partial \mathcal{M}_{(1,1)}=\left\{P, P^{\perp}: P=\left[L^{2}(E)\right], E=[-a, a], a \in[0, \infty]\right\}
$$

and if $\beta=(1,1)-\alpha$ then

$$
\partial \mathcal{M}_{\beta}=\left\{P M_{\chi_{(0, \infty)}}+P^{\perp} M_{\chi_{(-\infty, 0)}}: P \in \partial \mathcal{M}_{\alpha}\right\} .
$$

We now examine the order structure on $\Sigma_{m, n}$, which is rather simple in many cases.

Lemma 5.7. Suppose that $u(x)=\exp \left(i\left(\gamma x^{m}+\delta x^{n}\right)\right)$ is an inner function, where $n, m$ are distinct integers and $\gamma, \delta \in \mathbb{R}$. If $\gamma \neq 0$ then $m \in\{-1,0,1\}$, and if $\delta \neq 0$ then $n \in\{-1,0,1\}$.

Proof. Given $h(x) \in H^{\infty}(\mathbb{R})$, there is a unique function, $\eta(z) \in H^{\infty}(\mathbb{H})$ whose nontangential limit boundary value function $\eta^{\star} \in H^{\infty}(\mathbb{R})$ is equal to $h$ almost everywhere. Suppose that $\kappa(z)$ is analytic on an open $\operatorname{disc} U$ with $U \cap \mathbb{R}=(a, b)$ for some $a<b$, and that $h$ agrees with $\kappa^{\star}$ almost everywhere on $(a, b)$. Then $\eta(z)=\kappa(z)$ on $U \cap \mathbb{H}$. Indeed $\eta$ and $\kappa$ both have restrictions in $H^{\infty}(U \cap \mathbb{H})$ and their boundary functions agree on a set of positive measure, so the conclusion follows from the 
F. \& M. Riesz theorem together with the Riemann mapping theorem. (See also Fisher [5].)

We apply this principle to $h(x)=\exp \left(i\left(\gamma x^{m}+\delta x^{n}\right)\right)$. If $h$ is inner and $\eta \in H^{\infty}(\mathbb{H})$ with $\eta^{\star}=h$, consider the analytic function $\kappa: \mathbb{C} \backslash\{0\} \rightarrow \mathbb{C}$, $z \mapsto \exp \left(i\left(\gamma z^{m}+\delta z^{n}\right)\right)$. Since $\kappa$ is analytic on each of the open $\operatorname{discs} U$ which meet the right half line and the union of these discs contains $\mathbb{H}$, we conclude that $\eta=\kappa$ on $\mathbb{H}$. However, it is routine to check that this function is bounded in the upper half plane only under the stated conditions.

Lemma 5.8. Let $m, n$ be distinct integers in $\mathbb{Z} \backslash\{-1,0,1\}$. If $P$ and $Q$ are distinct projections in $\Sigma_{m, n}$ with $0 \neq P \leq Q \neq I$ then they must lie in $\partial \mathcal{M}_{m, n}$.

Proof. Suppose that $P \notin \partial \mathcal{M}_{m, n}$. By the F. \& M. Riesz theorem, $Q \notin \partial \mathcal{M}_{m, n}$. Suppose without loss of generality that $P \in \mathcal{M}_{m, n}$; if this is not the case, then apply the unitary automorphism $J_{2}$ induced by $x \mapsto-x$, which maps $\mathcal{M}_{m, n}^{\perp}$ onto $\mathcal{M}_{m, n}$, to make it so. If $Q \in \mathcal{M}_{m, n}^{\perp}$ then there exist $\alpha, \beta, \lambda, \mu \in \mathbb{R}$ such that

$$
e^{i\left(\lambda x^{m}+\mu x^{n}\right)} H^{2}(\mathbb{R}) \subseteq e^{i\left(\alpha x^{m}+\beta x^{n}\right)} \overline{H^{2}(\mathbb{R})},
$$

where these subspaces are the ranges of $P$ and $Q$, respectively. Let $u(x)=\exp i\left(\gamma x^{m}+\delta x^{n}\right)$ where $\gamma=\lambda-\alpha$ and $\delta=\mu-\beta$, so that $u H^{2}(\mathbb{R}) \subseteq \overline{H^{2}(\mathbb{R})}$. Taking complex conjugates yields $\bar{u} \overline{H^{2}(\mathbb{R})} \subseteq H^{2}(\mathbb{R})$, so $\overline{H^{2}(\mathbb{R})} \subseteq u H^{2}(\mathbb{R})$ and thus $u H^{2}(\mathbb{R})=\overline{H^{2}(\mathbb{R})}$. So

$$
H^{2}(\mathbb{R})=\left(\overline{H^{2}(\mathbb{R})}\right)^{\perp}=\left(u H^{2}(\mathbb{R})\right)^{\perp}=u \overline{H^{2}(\mathbb{R})}=u^{2} H^{2}(\mathbb{R}) .
$$

It is well-known that a unimodular function which preserves $H^{2}(\mathbb{R})$ must be constant almost everywhere, so $\gamma=\delta=0$, which would imply that $H^{2}(\mathbb{R})=\overline{H^{2}(\mathbb{R})}$, an obvious contradiction.

So $Q \in \mathcal{M}_{m, n}$, say

$$
P=\left[e^{i\left(\lambda x^{m}+\mu x^{n}\right)} H^{2}(\mathbb{R})\right], \quad Q=\left[e^{i\left(\alpha x^{m}+\beta x^{n}\right)} H^{2}(\mathbb{R})\right] .
$$

Now $u(x)=\exp i\left(\gamma x^{m}+\delta x^{n}\right)$ leaves $H^{2}(\mathbb{R})$ invariant, so is an inner function. Hence $\gamma=\delta=0$ by Lemma 5.7 and $P=Q$, a contradiction.

So $P \in \partial \mathcal{M}_{m, n}$, hence $Q \in \partial \mathcal{M}_{m, n}$ by the F. \& M. Riesz theorem.

The conclusion of Lemma 5.8 holds nontrivially precisely when $m, n$ are not both even and it follows that the boundary $\partial \mathcal{M}_{m, n}$ is a unitary invariant for $\Sigma_{m, n}$ and also for the balls $\mathcal{M}_{m, n}$. Furthermore, Lemma 5.6 shows that $\partial \mathcal{M}_{m, n}$ generates $L^{\infty}(\mathbb{R})$ precisely when $m, n$ are not both odd. When both these conditions prevail we can classify the spheres and balls by an argument similar to that of Theorem 5.5. In fact we expect that a somewhat deeper analysis will show that in general the (unordered) set

$$
\{\{m, n\},\{-m,-n\}\}
$$


is a complete unitary invariant.

We shall need the following elementary lemma.

Lemma 5.9. Let $m, n, p, q$ be nonzero integers with $m, n \geq 1$ such that $p \neq q$ and $m \neq n$. If $\gamma: \mathbb{R} \rightarrow \mathbb{R}$ induces a conformal automorphism of the upper half plane and $\alpha, \beta$ are real constants such that

$$
x^{p}-x^{q}=\alpha \gamma(x)^{m}+\beta \gamma(x)^{n}+2 \pi N(x) \quad \text { almost everywhere, }
$$

where $N: \mathbb{R} \rightarrow \mathbb{Z}$ then

$$
\{\{m, n\},\{-m,-n\}\}=\{\{p, q\},\{-p,-q\}\} .
$$

Proof. Without loss of generality, suppose that $p>q$ and $m>n$. Either $\gamma(x)=a x+b$ where $a>0$ and $b \in \mathbb{R}$, or $\gamma(x)=a-b(x-c)^{-1}$ for $a, c \in \mathbb{R}$ and $b>0$. Suppose first that $\gamma(x)=a x+b$; without loss of generality, we may take $a=1$. Since $N$ is then continuous and so constant on $(0, \infty)$ it follows that $p, q \geq 1$ and so $N$ is constant on $\mathbb{R}$. The equation

$$
x^{p}-x^{q}=\alpha(x+b)^{m}+\beta(x+b)^{n}+2 \pi N
$$

holds almost everywhere. Considering the coefficient of $x^{p}$ gives $\alpha=1$ and $m=p$, so we suppose that $n \neq q$. Differentiating gives

$$
p x^{p-1}-q x^{q-1}=p(x+b)^{p-1}+\beta n(x+b)^{n-1} .
$$

If $q, n>1$ then we set $x=-b$ to deduce that $b$ is algebraic, and set $x$ equal to any other algebraic number to see that $\beta$ is also algebraic. Simple arguments show that the same holds if $q>1$ and $n=1$ or if $q=1$ and $n>1$. Now equate the constant terms in the original expression:

$$
2 \pi N=-\left(b^{p}+\beta b^{n}\right) .
$$

Since $N \in \mathbb{Z}$ and the right hand side is algebraic, $N=0$. By counting repeated roots, it now follows that $n=q$.

If on the other hand $\gamma(x)=a-b(x-c)^{-1}$ then $N$ is continuous and so constant on the components of $\mathbb{R} \backslash\{0, c\}$, so $p, q \leq-1$ and $N(x)=0$ almost everywhere for $x>\max \{0, c\}$ and for $x<\min \{0, c\}$. Since the left hand side is locally unbounded only at $x=0$ and has limit 0 as $x \rightarrow \pm \infty$, we must have $c=0$ and $N(x)=0$ almost everywhere. It only remains to consider the order of growth and decay at 0 and $\pm \infty$ to see that $p=-n$ and $q=-m$.

Theorem 5.10. Let $p, q \in \mathbb{Z} \backslash\{-1,0,1\}$ with $p \neq q$ and let $m, n>1$ be integers with $m \not \equiv n \bmod 2$. The spheres $\Sigma_{m, n}$ and $\Sigma_{p, q}$ are unitarily equivalent if and only if

$$
\{\{m, n\},\{-m,-n\}\}=\{\{p, q\},\{-p,-q\}\} .
$$


Proof. First observe that the spheres are unitarily equivalent if these sets are equal, since the composition operator $J_{1}$ corresponding to the map $x \mapsto-x^{-1}$ satisfies $\left(\operatorname{Ad} J_{1}\right) \Sigma_{m, n}=\Sigma_{-m,-n}$.

Let $U \in \operatorname{Unit}\left(L^{2}(\mathbb{R})\right)$ with $(\operatorname{Ad} U) \Sigma_{m, n}=\Sigma_{p, q}$. Consider the subspace $U H^{2}(\mathbb{R}) \in \Sigma_{p, q}$. Since $\operatorname{Ad} U$ preserves the order structure, it must map $\partial \mathcal{M}_{m, n}$ onto $\partial \mathcal{M}_{p, q}$ by Lemmas 5.6 and 5.8. By composition with $x \mapsto-x$ if necessary, we may assume that $U H^{2}(\mathbb{R}) \in \mathcal{M}_{p, q}$ and then translating by the "obvious" inner automorphisms of $\mathcal{M}_{p, q}$, that $U H^{2}(\mathbb{R})=H^{2}(\mathbb{R})$.

Let $\sigma$ be the residue class of $(m, n)$ in $(\mathbb{Z} / 2 \mathbb{Z})^{2}$, which by assumption is in $\{(0,1),(1,0)\}$. Observe that by Lemma 5.6, the von Neumann algebra generated by $\partial \mathcal{M}_{m, n}=\partial \mathcal{M}_{\sigma}$ is the multiplication algebra $L^{\infty}(\mathbb{R})$, and the only possibilities for the algebra $\mathcal{A}=\left(\partial \mathcal{M}_{p, q}\right)^{\prime \prime}$ are

$$
\mathcal{A}=L^{\infty}(\mathbb{R}) \quad \text { or } \quad \mathcal{A}=\left\{M_{f}: f \in L^{\infty}(\mathbb{R}), f(x)=f(-x)\right\} .
$$

The latter algebra has uniform multiplicity 2. Since $\operatorname{Ad} U$ sends projections in $\left(\partial \mathcal{M}_{m, n}\right)^{\prime \prime}$ to projections in $\left(\partial \mathcal{M}_{p, q}\right)^{\prime \prime}$, it induces an isomorphism between $L^{\infty}(\mathbb{R})$ and $\mathcal{A}$. Spatial isomorphisms preserve multiplicity, so in fact $\mathcal{A}=L^{\infty}(\mathbb{R})$.

Now $\operatorname{Ad} U$ is an isomorphism $L^{\infty}(\mathbb{R}) \rightarrow L^{\infty}(\mathbb{R})$ and it follows that $U=M_{\varphi} C_{\gamma}$ where $\varphi \in L^{\infty}(\mathbb{R})$ is unimodular and $C_{\gamma}$ is a unitary composition operator with symbol $\gamma$, a Borel isomorphism. Exactly as in the proof of Theorem 5.5, $\gamma$ induces a conformal automorphism of the upper half plane. Since $\operatorname{Ad} U$ is a homeomorphism with

$$
U H^{2}(\mathbb{R})=H^{2}(\mathbb{R}) \quad \text { and } \quad U \partial \mathcal{M}_{m, n}=\partial \mathcal{M}_{p, q},
$$

it maps the two components $\mathcal{M}_{m, n}$ and $\mathcal{M}_{m, n}^{\perp}$ of $\Sigma_{m, n} \backslash \partial \mathcal{M}_{m, n}$ to $\mathcal{M}_{p, q}$ and $\mathcal{M}_{p, q}^{\perp}$ respectively. In particular, there exist real $\alpha, \beta$ such that

$$
\begin{aligned}
e^{i\left(x^{p}-x^{q}\right)} H^{2}(\mathbb{R}) & =U e^{i\left(\alpha x^{m}+\beta x^{n}\right)} H^{2}(\mathbb{R}) \\
& =e^{i\left(\alpha \gamma(x)^{m}+\beta \gamma(x)^{n}\right)} U H^{2}(\mathbb{R}) \\
& =e^{i\left(\alpha \gamma(x)^{m}+\beta \gamma(x)^{n}\right)} H^{2}(\mathbb{R}) .
\end{aligned}
$$

Hence the hypotheses of Lemma 5.9 are satisfied and the result follows.

\section{REFERENCES}

[1] M. J. Cowen and R. G. Douglas. Complex geometry and operator theory. Acta Math., 141(3-4):187-261, 1978.

[2] R. E. Curto and N. Salinas. Generalized Bergman kernels and the CowenDouglas theory. Amer. J. Math., 106(2):447-488, 1984.

[3] K. R. Davidson. Nest algebras, volume 191 of Pitman Research Notes in Mathematics Series. Longman Scientific \& Technical, Harlow, 1988.

[4] R. G. Douglas. Banach algebra techniques in operator theory, volume 236 of Graduate Texts in Mathematics. Springer, New York, second edition, 1998.

[5] S. D. Fisher. Function theory on planar domains. Dover Publications Inc., New York, 2007. 
[6] J. B. Garnett. Bounded analytic functions, volume 236 of Graduate Texts in Mathematics. Springer, New York, revised first edition, 2007.

[7] A. Katavolos and S. C. Power. The Fourier binest algebra. Math. Proc. Camb. Phil. Soc., 122(3):525-539, 1997.

[8] A. Katavolos and S. C. Power. Translation and dilation invariant subspaces of $L^{2}(\mathbb{R})$. J. Reine Angew. Math., 552:101-129, 2002.

[9] S. Lang. $S L_{2}(\mathbb{R})$, volume 105 of Graduate Texts in Mathematics. SpringerVerlag, New York, 1985. Reprint of the 1975 edition.

[10] P. D. Lax. Translation invariant spaces. Acta Math., 101:163-178, 1959.

[11] R. H. Levene. Lie semigroup operator algebras. PhD thesis, Lancaster University, 2004.

[12] R. H. Levene and S. C. Power. Reflexivity of the translation-dilation algebras on $L^{2}(\mathbb{R})$. Int. J. Math., 14(10):1081-1090, 2003.

[13] N. K. Nikolski. Operators, functions, and systems: an easy reading. Vol. 1, volume 92 of Mathematical Surveys and Monographs. American Mathematical Society, Providence, RI, 2002. Hardy, Hankel, and Toeplitz, Translated from the French by Andreas Hartmann.

[14] E. A. Nordgren, P. Rosenthal, and F. S. Wintrobe. Composition operators and the invariant subspace problem. C. R. Math. Rep. Acad. Sci. Canada, 6(5):279-283, 1984.

[15] S. C. Power. Completely contractive representations for some doubly generated antisymmetric operator algebras. Proc. Amer. Math. Soc., 126(8):2355-2359, 1998.

[16] S. C. Power. Invariant subspaces of translation semigroups. In Proceedings of the First Advanced Course in Operator Theory and Complex Analysis, pages 37-50. Universidad de Sevilla, 2006.

[17] E. M. Stein. Harmonic analysis: real-variable methods, orthogonality, and oscillatory integrals, volume 43 of Princeton Mathematical Series. Princeton University Press, Princeton, NJ, 1993.

[18] M. E. Taylor. Noncommutative harmonic analysis, volume 22 of Mathematical Surveys and Monographs. American Mathematical Society, Providence, RI, 1986.

[19] J. E. Thomson. Invariant subspaces for subnormal operators. In Surveys of some recent results in operator theory, Vol. I, volume 171 of Pitman Res. Notes Math. Ser., pages 241-259. Longman Sci. Tech., Harlow, 1988.

[20] J. von Neumann. Einige Sätze über messbare Abbildungen. Ann. of Math. (2), $33(3): 574-586,1932$.

[21] K. Zhu. Operator theory in function spaces, volume 138 of Mathematical Surveys and Monographs. American Mathematical Society, Providence, RI, second edition, 2007.

School of Mathematics, Trinity College Dublin, Ireland

E-mail address: levene@maths.tcd.ie

Department of Mathematics and Statistics, Lancaster University, LANCASTER LA1 4YF, UK

E-mail address: s.power@lancaster.ac.uk 